\title{
A geometric higher twist effect on nuclear target
}

\author{
Wei Zhu \\ Department of Physics, East China Normal University, Shanghai 200062, P.R. China
}

\begin{abstract}
The higher twist effects in deep inelastic scattering on the nuclear target are studied using time ordered perturbation theory. We showed that the collinear rescattering of the outgoing quark on the extra nucleons via the contacting gluon-pair is dominant in nuclear size-dependent effects. The Qiu-Vitev resummation is proved by using the geometric properties of the higher twist amplitudes. The leading contributions of nuclear-enhanced effect to the DGLAP evolution equation are resummed in the same framework.
\end{abstract}

PACS numbers: 13.60.Hb; 12.38.Bx.

keywords: Higher twist effect; PQCD evolution equation 


\section{Introduction}

Deep inelastic scattering (DIS) experiments are the main source extracting the information about QCD structure of the hadronic target. The DIS amplitudes can be expanded according to their twists. The leading-twist amplitudes describe the quark-gluon structure of a target at short distance, while the higher twist effects and their summation may provide a valuable information for the multi-parton correlations at long distance in the target [1]. The nuclear target is an ideal "laboratory" for testing higher twist theory because of its special multi-parton configuration.

However, it is difficult to sum all contributions in the twist expansion due to the complicated structure of the higher twist amplitudes and the unknown multi-parton correlation functions. Instead of a complete summation, the resummation of the leading contributions to a special effect from the twist expansion is useful. For example, the Glauber model is used to resum the contributions of the multi-parton scattering to the shadowing effect in the nuclear structure functions in the Glauber-Mueller formula [2]. This formula assumes that the leading contributions are from the incoherent scattering of color dipole on the nuclear gluons. Another example in this realm is the JalilianMarian-Iancu-McLerran-Weigert-Leonidov-Kovner (JIMWLK) equation [3], where the soft nuclear gluons are treated as a classical color potential because of its large density at small $x_{B}$. In this background, energetic partons in the multi-dipole configuration interact with soft gluons through the Wilson lines, which resum the contributions of the soft nuclear gluons at the eikonal approximation $[4,5]$. Both of the above two researches are broadly used to predict the saturation phenomena in the nuclear structure functions, where unitarity is restored. 
On the other hand, a different resummation of the nuclear higher twist amplitudes is recently proposed by Qiu and Vitev (Qiu-Vitev resummation) [6]. This model assumes that the leading contributions to the nuclear size effect in the structure functions originate from the singularities of the gluon correlation functions in the higher twist amplitudes [7].

The higher twist amplitudes have complicated color-spin structure. The resummation of a higher twist effect is valid only if the contributions from a lot of irrelevant diagrams can be entirely neglected. Unfortunately, this point is unclear in the researches mentioned above.

The motivation of the present work is to study the geometric properties of the higher twist amplitudes for the DIS on nuclear target using time ordered perturbation theory (TOPT) [8], which was developed in the collinear approximation (CLTOPT) in [9]. Comparing with the standard covariant perturbation theory (CVPT), the CLTOPT provides a more intuitive geometric character of the higher twist amplitudes. Using the above TOPT method we study the DIS processes on the nuclear target at arbitrary twist. We present a geometric higher twist effect in the DIS on the nuclear target: the leading contributions to a nuclear size-dependent effect in the DIS processes originate from the multiple rescattering of the outgoing quark on the extra nucleon via the exchange of the contacting gluon-pair. It reproduces the Qiu-Vitev resummation [6] but using different dynamics. In particular, this space-time character of the amplitudes in the CLTOPT allows us to distinguish the leading diagrams from a lot of sub-leading diagrams without calculations of the Feynman diagrams. We resum the corrections of the geometric higher twist effect to the structure function and DGLAP evolution equation $[10,11]$.

The paper is organized as follows. In Section 2 we present a short review about 
TOPT in the collinear approximation, which is necessary for understanding this work. For a complete calculation of the higher twist amplitude including the matrix element, we discuss in Section 3 the normalization of the correlation functions. In Section 4, using CLTOPT we demonstrate the space-time picture of the higher twist amplitudes, which exposes obvious nuclear size-dependence. In Section 5 we derive the expressions for the nuclear dependence of the structure function at twist-4 level, then the results are generalized to sum the contributions of all twist amplitudes. In Section 6 we study the corrections of the above mentioned higher twist effect to the DGLAP evolution equation. The discussions and a summary are presented in Section 7 . 


\section{Definitions and tools}

We consider DIS of leptons from an unpolarized nuclear target. We denote the lightlike vectors along the positive and negative directions as $n^{\mu} \equiv 1 / \sqrt{2}\left(1,0_{\perp},-1\right)$ and $\bar{n}^{\mu} \equiv$ $1 / \sqrt{2}\left(1,0_{\perp}, 1\right)$. Therefore, we have

$$
n \cdot n=\bar{n} \cdot \bar{n}=0, \quad \bar{n} \cdot n=1
$$

As is well known, the infinite momentum frame is equivalent to the light-cone coordinate. Here, however, we would rather use TOPT in the infinite momentum frame than the light-cone quantization. The reason is that the contributions of the backward (instantaneous) propagator in the latter case are neglected and it is not a complete description for the higher twist processes. In the concrete, we choose an infinite frame, in which the target momentum and virtual photon momentum have the following forms,

$$
\begin{aligned}
P^{\mu} & =P^{+} \bar{n}^{\mu} \\
q^{\mu} & =q^{+} \bar{n}+q^{-} n=-x_{B} P^{+} \bar{n}^{\mu}+\frac{Q^{2}}{2 x_{B} P^{+}} n^{\mu},
\end{aligned}
$$

where $Q^{2}=-q^{2}$ and $x_{B}$ is the Bjorken variable.

In this work we take the physical axial gauge: $n \cdot A=0$, with $A$ being the gluon field. The inclusive cross-section for the unpolarized DIS is determined by the hadronic tensor $W^{\mu \nu}$, which can be decomposed into the transverse structure function $F_{T}\left(x_{B}, Q^{2}\right)$ and longitudinal one $F_{L}\left(x_{B}, Q^{2}\right)$,

$$
W^{\mu \nu}\left(x_{B}, Q^{2}\right)=e_{L}^{\mu \nu} F_{L}\left(x_{B}, Q^{2}\right)+e_{T}^{\mu \nu} F_{T}\left(x_{B}, Q^{2}\right)
$$


The projection operators are defined as

$$
\begin{aligned}
e_{L}^{\mu \nu} & =\frac{1}{2 Q^{2}}\left(x_{B} P^{+} \bar{n}^{\mu}+\frac{Q^{2}}{2 x_{B} P^{+}} n^{\mu}\right)\left(x_{B} P^{+} \bar{n}^{\nu}+\frac{Q^{2}}{2 x_{B} P^{+}} n^{\nu}\right) \\
e_{T}^{\mu \nu} & =\frac{1}{2}\left(\bar{n}^{\mu} n^{\nu}+\bar{n}^{\nu} n^{\mu}-g^{\mu \nu}\right) \equiv \frac{1}{2} d^{\mu \nu} .
\end{aligned}
$$

Clearly, using

$$
e_{T}^{\mu \nu} \cdot e_{T}^{\mu \nu}=\frac{1}{2}, \quad e_{L}^{\mu \nu} \cdot e_{L}^{\mu \nu}=\frac{1}{4} \text { and } e_{T}^{\mu \nu} \cdot e_{L}^{\mu \nu}=0
$$

one can directly extract $F_{T}\left(x_{B}, Q^{2}\right)$ and $F_{L}\left(x_{B}, Q^{2}\right)$ from $W^{\mu \nu}$ according to

$$
\begin{aligned}
& F_{T}\left(x_{B}, Q^{2}\right)=2 e_{T}^{\mu \nu} W^{\mu \nu}\left(x_{B}, Q^{2}\right), \\
& F_{L}\left(x_{B}, Q^{2}\right)=4 e_{L}^{\mu \nu} W^{\mu \nu}\left(x_{B}, Q^{2}\right) .
\end{aligned}
$$

Note that the hadronic tensor $W^{\mu \nu}\left(x_{B}, Q^{2}\right)$ can also be decomposed into the structure functions $F_{1}\left(x_{B}, Q^{2}\right)$ and $F_{2}\left(x_{B}, Q^{2}\right)$ and their relations with Eqs. (7) and (8) are

$$
\begin{aligned}
& F_{L}\left(x_{B}, Q^{2}\right)=\frac{F_{2}\left(x_{B}, Q^{2}\right)}{x_{B}}-2 F_{1}\left(x_{B}, Q^{2}\right), \\
& F_{T}\left(x_{B}, Q^{2}\right)=2 F_{1}\left(x_{B}, Q^{2}\right) .
\end{aligned}
$$

To further examine the role of higher twist corrections in the DIS processes, we present the TOPT-form of the QCD-propagators in the collinear limit [9]. As will be discussed in details below, the nuclear dependence on both the structure functions and evolution equations is dominated by the higher twist amplitudes. A straightforward reason is that the amplitude at higher twist contains the multi-parton operators in the matrix element, which can come from the different nucleons of a nucleus and give rise to the nuclear 
size-dependence. However, we have a lot of amplitudes corresponding to the multiple interactions among the partons. The important question is: which of them contributes the leading nuclear effect to the processes at a given order of approximation? We find that the TOPT in the collinear limit, which is developed in our previous works [9], is a useful tool for answering the above question.

TOPT is exactly equivalent to the method of the Feynman diagram [1]. A covariant propagator in the Feynman diagram is decomposed to the forward- and backwardpropagators in TOPT and this leads to two remarkable properties: the interaction is ordered on time and the propagating momentum is on the mass shell. As we will point out in the following, the former shows how a process evolves in the light-cone coordinates and gives us information about the nuclear effects, while the latter is useful to decompose a complicated calculation.

At first sight it seems like that TOPT leads to a proliferation of diagrams. However, we find that there is only one TOPT diagram corresponding to one Feynman diagram in the collinear approximation, where the transverse momentum of the parton is neglected. In fact, the TOPT-propagators in the collinear limit have the following special forms [9]

$$
\begin{aligned}
S_{F}(k) & =\frac{\gamma \cdot \bar{n}}{2 k \cdot \bar{n}} \\
S_{B}(k) & =\frac{\gamma \cdot n}{2 k \cdot n}
\end{aligned}
$$

for quarks, and

$$
\begin{aligned}
G_{F}^{\alpha \beta}(k) & =\frac{d_{\perp}^{\alpha \beta}}{k \cdot \bar{n}(k \cdot n-k \cdot \bar{n})} \\
G_{B}^{\alpha \beta}(k) & =\frac{n^{\alpha} n^{\beta}}{(k \cdot n)^{2}},
\end{aligned}
$$


for gluons, where " $F$ " and " $B$ " label the forward- and backward-moving partons, respectively. Using helicity conservation and Eq. (1), the non-vanishing vertices of QCD and QED in the collinear approximation are shown in Figs. 1 and 2, respectively. According to those selection rules, we draw the twist- 4 amplitudes, which contribute to $F_{T}\left(x_{B}, Q^{2}\right)$ and $F_{L}\left(x_{B}, Q^{2}\right)$ in the collinear limit, in Figs. 3 and 4, where we label the outgoing quark or outgoing gluon as " $B$ " or " $F$ ", since it contributes $\sim \gamma \cdot n$ or $\sim d_{\perp}^{\alpha \beta}$, respectively. We did not draw the conjugate diagrams. One finds that only one TOPT graph corresponds to one covariant Feynman diagram in the collinear limit.

The deep inelastic interaction in the TOPT-form is time-ordered in a given infinite momentum frame and shows how a system evolves in the light-cone coordinates. For illustrating this picture, we take the Fourier transformation of the forward- and backwardpropagators. Note that the right hand sides of Eqs. (10) and (11) indicate that $S_{F}$ is only a function of $k^{-}$, while $S_{B}$ is related to $k^{+}$. Thus, we have

$$
\begin{aligned}
\int S_{F}\left(k^{-}\right) \exp \left[i k \cdot\left(y_{1}-y_{2}\right)\right] \frac{d^{4} k}{(2 \pi)^{4}} & =f_{F}\left(y_{1}^{+}-y_{2}^{+}\right) \delta\left(y_{1}^{-}-y_{2}^{-}\right) \delta^{(2)}\left(y_{1}^{\perp}-y_{2}^{\perp}\right) \\
& \equiv S_{F}\left(y_{1}^{+}-y_{2}^{+}\right), \\
\int S_{B}\left(k^{+}\right) \exp \left[i k \cdot\left(y_{1}-y_{2}\right)\right] \frac{d^{4} k}{(2 \pi)^{4}} & =f_{B}\left(y_{1}^{-}-y_{2}^{-}\right) \delta\left(y_{1}^{+}-y_{2}^{+}\right) \delta^{(2)}\left(y_{1}^{\perp}-y_{2}^{\perp}\right) \\
& \equiv S_{B}\left(y_{1}^{-}-y_{2}^{-}\right) .
\end{aligned}
$$

These expressions show that the forward- and backward-propagators for quarks in the collinear approximation are parallel to $y^{+}$- and $y^{-}$-axes, respectively. Using this property, for example, we draw the twist-4 amplitudes of Fig. 3a in the $y^{+}-y^{-}$-coordinate in Fig. 5a. One finds that $S_{F}$ and $S_{B}$ correspond to the contact- and instantaneous-interactions 
in the light-cone coordinates. The backward propagator of the gluon (Eq. (13)) has the same property as $S_{B}$, i.e., $G_{B}\left(y_{1}^{-}-y_{2}^{-}\right)$is always parallel to the $y^{-}$-axis. We focus on the relation between the interactions of $\gamma^{*}$-parton and the structure of the correlation functions, which are defined on the $y^{-}$-axis in the collinear factorization. Therefore, for simplicity, we project the interactions of $\gamma^{*}$-parton in Fig. 5a on the $y^{-}$-axis (Fig. 5b). Thus, we get the geometric diagrams Figs. 6 and 7, corresponding to Figs, 3 and 4, respectively. Here we note that Eq. (12) indicates that $G_{F}(k)$ is a function of both $k^{-}$ and $k^{+}$. Therefore, the forward propagator of gluon has two components-along $y^{+}$and $y^{-}$- even in the collinear limit. An exception is that the gluon propagator connects with the hard- and soft-parts of the amplitude. The factorization scheme [12] considers that soft gluons attach to this propagator at the eikonal approximation. In this case, the largest "+" component $p^{+}$of the three-gluon vertex in the numerator shall cancel against a corresponding term in the denominator of the gluon propagator and the resulting $G_{F}$ only contains its $y^{+}$-component.

Similarly, we can draw the twist-6 amplitudes. There are many different TOPT diagrams. Some typical diagrams of them are shown in Figs. 8 and 9 for the transverse and longitudinal coefficient functions, respectively. Figures 10 and 11 are corresponding geometric TOPT diagrams. It is really difficult to judge which diagrams give leading contributions to the nuclear size-dependent effect in Figs. 3, 4, 8 and 9. However, we shall show that the leading corrections in the corresponding geometric diagrams only come from a few of diagrams (Figs. 6a, 10a and 10b), which contain maximum number of colorless gluon pair, and such corrections can be simply resummed. 


\section{Normalization condition}

Any calculation of the DIS cross section needs a factorization scheme to separate the calculable partonic part from the nonperturbative matrix element. In such a scheme, the propagators with the spinor trace or the Lorentz indices linking two separated parts are decomposed. At the same time, an arbitrary constant may sneak into the undefined correlation function in this technique. Therefore, at first we determine the normalization

constant in the definition of the correlation function. For this reason, we begin with the naive parton model, which defines the quark distribution functions $f_{q_{i}}\left(x_{B}\right)$. According to Fig. 12, the transverse structure function $F_{T}$ at the leading order takes the form,

$$
\begin{aligned}
F_{T}\left(x_{B}\right) & =2 e_{T}^{\mu \nu} W^{\mu \nu} \\
& =\sum_{i} e_{i}^{2} \int d x \frac{1}{2} \operatorname{Tr}\left[x P^{+} \gamma \cdot \bar{n} \gamma^{\mu} \frac{Q^{2}}{2 x_{B} P^{+}} \gamma \cdot n \gamma^{\nu}\right] d_{\perp}^{\mu \nu} \delta\left(x-x_{B}\right) \frac{x_{B}}{Q^{2}} T_{2 q}(x) \\
& =\sum_{i} 2 e_{i}^{2} x_{B} T_{2 q}\left(x_{B}\right) \equiv \sum_{i} e_{i}^{2} f_{q_{i}}\left(x_{B}\right),
\end{aligned}
$$

where we replace the propagator $\left(k^{\prime 2}+i \epsilon\right)^{-1}$ with $\delta\left(k^{\prime 2}\right)=x_{B} \delta\left(x-x_{B}\right) / Q^{2}$ due to the on-shell condition of the parton model. Thus, we have $f_{q_{i}}(x)=2 x T_{2 q}(x)$, where

$$
T_{2 q}(x)=\int \frac{P^{+} d y^{-}}{2 \pi} e^{i x P^{+} y^{-}}<N\left|\bar{\Psi}_{i}(0) \frac{\gamma^{+}}{2 P^{+}} \Psi_{i}\left(y^{-}\right)\right| N>
$$

The normalization constant in the gluon distribution function can be determined by using the contribution of gluons to $F_{T}\left(x_{B}, Q^{2}\right)$ at the leading logarithmic approximation (Fig. 13). In the TOPT form, we have

$$
F_{T}^{L L A}\left(x_{B}, Q^{2}\right)=d_{\perp}^{\mu \nu} W^{\mu \nu}
$$




$$
\begin{aligned}
= & \sum_{i} e_{i}^{2} \int \frac{d^{3} \vec{k}_{1}}{(2 \pi)^{3}}<\frac{1}{2}>_{\text {colour }} 4 \pi \alpha_{s} \frac{E_{p}}{E_{\hat{k}_{S}}} \frac{1}{8 E_{\hat{k}_{S}} E_{p} E_{k_{1}}}\left(\frac{1}{E_{\hat{k}_{S}}+E_{k_{1}}-E_{p}}\right)^{2} \\
& \times \frac{1}{2} \operatorname{Tr}\left[\gamma \cdot k_{1} \gamma^{\alpha} \gamma \cdot \hat{k}_{S} \gamma^{\beta}\right] d_{\perp}^{\alpha \beta} \operatorname{Tr}\left[\gamma^{\mu} \gamma \cdot k_{2} \gamma^{\nu} \gamma \cdot \hat{k}_{S}\right] d_{\perp}^{\mu \nu} \delta\left(z_{S}-\frac{x_{B}}{y}\right) \frac{x_{B}}{Q^{2}} T_{2 g} \\
\simeq & \sum_{i} e_{i}^{2} \int \frac{d k_{\perp}^{2}}{k_{\perp}^{2}} d z_{s} \frac{\alpha_{s}}{2 \pi}\left[\left(\frac{x_{B}}{y}\right)^{2}+\left(1-\left(\frac{x_{B}}{y}\right)^{2}\right)\right] f_{g}(y),
\end{aligned}
$$

where the momenta of partons in Fig.13 are parameterized as

$$
\begin{aligned}
p & =\left[y P^{+}, \underline{0}, y P^{+}\right], \\
\hat{k}_{S} & =\left[x P^{+}+\frac{k_{\perp}^{2}}{2 x P^{+}}, k_{\perp}, x P^{+}\right], \\
k_{1} & =\left[(1-x) P^{+}+\frac{k_{\perp}^{2}}{2(1-x) P^{+}},-k_{\perp},(1-x) P^{+}\right] .
\end{aligned}
$$

The definition of $T_{2 g}(x)$ in Eq. (18) is

$$
T_{2 g}(x)=\int \frac{P^{+} d y^{-}}{2 \pi} e^{i x P^{+} y^{-}}<N\left|A^{+\alpha}(0) A^{+\beta}\left(y^{-}\right)\right| N>d_{\perp}^{\alpha \beta}
$$

or

$$
T_{2 g}(x)=\int \frac{P^{+} d y^{-}}{2 \pi} e^{i x P^{+} y^{-}}<N\left|F^{+\alpha}(0) F^{+\beta}\left(y^{-}\right)\right| N>\left(\frac{1}{x P^{+}}\right)^{2} d_{\perp}^{\alpha \beta}
$$

where $A^{+\alpha} \rightarrow F^{\alpha \beta} n^{\beta} /\left(x P^{+}\right)$and $F^{\alpha \beta}$ is the gluon field strength. The second definition has the gauge invariant form and presents singularity when $x \rightarrow 0$, which implies the exchange of soft gluon between the nonperturbative matrix and perturbative coefficient 
function. However, all those soft gluons should be absorbed into the definition of parton distribution functions (PDFs) in the collinear factorization scheme. Therefore, the factor $1 / x$ in Eq. (21) should not give rise to any observable effects in the perturbation part of the amplitude. For avoiding misunderstanding, it is different from Ref. [6], we use the gluon distribution function

$$
f_{g}(x)=\int \frac{P^{+} d y^{-}}{2 \pi} e^{i x P^{+} y^{-}}<N\left|A^{+\alpha}(0) A^{+\beta}\left(y^{-}\right)\right| N>d_{\perp}^{\alpha \beta} .
$$




\section{Geometric nuclear enhancement dynamics}

The nuclear target provides a special "laboratory" for the resummation of the higher twist effects. The contributions of the nuclear effects to the inclusive deep inelastic cross section are governed by the hadronic tensor. In the factorization scheme, the operator product in the hadronic tensor is expanded on the twist, where the higher twist amplitudes include the contributions of the multi-partonic configurations in the target. The contributions of the partons from different nucleons may give rise to a visible nuclear dependence in the structure functions.

The nuclear effects in the structure functions originate from the correlations of partons among the bound nucleons. The parton correlation function is a generalization of the parton density beyond the leading twist. A general correlation function for two quarks and two gluons is defined as

$$
\begin{aligned}
T_{2 q 2 g}= & \int \frac{P^{+} d y^{-}}{2 \pi} \frac{P^{+} d y_{1}^{-}}{2 \pi} \frac{P^{+} d y_{2}^{-}}{2 \pi} e^{i x_{1} P^{+} y^{-}} e^{i\left(x-x_{1}\right) P^{+} y_{1}^{-}} e^{-i\left(x-x_{2}\right) P^{+} y_{2}^{-}} \\
& \times \frac{1}{2 P^{+}} \frac{1}{A}<A\left|\bar{\Psi}\left(0^{-}\right) \gamma^{+} A^{+\alpha}\left(y_{1}^{-}\right) A^{+\beta}\left(y_{2}^{-}\right) \Psi\left(y^{-}\right)\right| A>d_{\perp}^{\alpha \beta}
\end{aligned}
$$

We always compare the structure function per bounded nucleon with that of a free nucleon to present the nuclear effects. Therefore, we do not expect a large nuclear dependence in the leading twist process, since

$$
\begin{aligned}
T_{2 q} & =\int \frac{P^{+} d y^{-}}{2 \pi} e^{i x P^{+} y^{-}} \frac{1}{2 P^{+}} \frac{1}{A}<A\left|\bar{\Psi}(0) \gamma^{+} \Psi\left(y^{-}\right)\right| A> \\
& \simeq \int \frac{P^{+} d y^{-}}{2 \pi} e^{i x P^{+} y^{-}} \frac{1}{2 P^{+}}<N\left|\bar{\Psi}(0) \gamma^{+} \Psi\left(y^{-}\right)\right| N>
\end{aligned}
$$

where $\mid A>$ and $\mid N>$ are the state vectors for nucleus and free nucleon, respectively. 
In the general case, the exponential oscillations in the higher twist matrix element destroy any nuclear size enhancement that could come from the $y^{-}$integration $[7,13]$. The contributions of the integrations with respect to $y_{1}^{-}$and $y_{2}^{-}$to Eq. (23) are namely from the gluonic fields neighboring the targeted quarks. In this case, we assume that

$$
\begin{array}{r}
\int \frac{P^{+} d y^{-}}{2 \pi} \frac{P^{+} d y_{1}^{-}}{2 \pi} \frac{P^{+} d y_{2}^{-}}{2 \pi} e^{i x_{1} P^{+} y^{-}} e^{i\left(x-x_{1}\right) P^{+} y_{1}^{-}} e^{-i\left(x-x_{2}\right) P^{+} y_{2}^{-}} \frac{1}{2 P^{+}} \\
\times \frac{1}{A}<A\left|\bar{\Psi}\left(0^{-}\right) \gamma^{+} A^{+\alpha}\left(y_{1}^{-}\right) A^{+\beta}\left(y_{2}^{-}\right) \Psi\left(y^{-}\right)\right| A>d_{\perp}^{\alpha \beta} \\
\simeq \int \frac{P^{+} d y^{-}}{2 \pi} \frac{P^{+} d y_{1}^{-}}{2 \pi} \frac{P^{+} d y_{2}^{-}}{2 \pi} e^{i x_{1} P^{+} y^{-}} e^{i\left(x-x_{1}\right) P^{+} y_{1}^{-}} e^{-i\left(x-x_{2}\right) P^{+} y_{2}^{-}} \frac{1}{2 P^{+}} \\
\times<N\left|\bar{\Psi}\left(0^{-}\right) \gamma^{+} A^{+\alpha}\left(y_{1}^{-}\right) A^{+\beta}\left(y_{2}^{-}\right) \Psi\left(y^{-}\right)\right| N>d_{\perp}^{\alpha \beta} .
\end{array}
$$

Therefore, we do not expect a nuclear size effect in Eq. (25). However, some TOPTdiagrams for $F_{T}\left(x_{B}, Q^{2}\right)$ at twist-4 (Fig. 14) show that $y_{1}^{-}=y_{2}^{-}$. We further consider the case where two gluonic operators consist a colorless cluster, which belongs to the same extra nucleon. In this case there is no strong correlation between the struck nucleon and the extra nucleon. The momentum exchange among the bounded nucleons can be neglected in comparison with a lager $P^{+}$-scale. Thus, we assume that $x_{1} \simeq x_{2}$ in Eq. (25). Now all nucleons listing on the integral path can contribute their gluons to the double scattering in Fig. 14 without distortion. Thus, we can write

$$
\begin{aligned}
T_{2 q 2 g}= & \int \frac{P^{+} d y^{-}}{2 \pi} \frac{P^{+} d y_{1}^{-}}{2 \pi} e^{i x_{1} P^{+} y^{-}} \frac{1}{A} \frac{1}{2 P^{+}} \eta_{\perp} \\
& \times<A\left|\bar{\Psi}\left(0^{-}\right) \gamma^{+} \Psi\left(y^{-}\right)\right| A><A\left|A^{+\alpha}\left(y_{1}^{-}\right) A^{+\beta}\left(y_{1}^{-}\right)\right| A>d_{\perp}^{\alpha \beta} \\
\simeq & \int \frac{P^{+} d y^{-}}{2 \pi} \frac{P^{+} d y_{1}^{-}}{2 \pi} e^{i x_{1} P^{+} y^{-}} \frac{1}{2 P^{+}} \eta_{\perp}<N\left|\bar{\Psi}\left(0^{-}\right) \gamma^{+} \Psi\left(y^{-}\right)\right| N> \\
& \times<A\left|A^{+\alpha}\left(y_{1}^{-}\right) A^{+\beta}\left(y_{1}^{-}\right)\right| A>d_{\perp}^{\alpha \beta}
\end{aligned}
$$




$$
\begin{aligned}
& =\eta_{\perp} \eta_{\|} T_{2 q} \Theta \\
& =\frac{A^{1 / 3}-1}{R^{2}} T_{2 q} \Theta
\end{aligned}
$$

where

$$
\Theta \equiv \int \frac{P^{+} d y_{1}^{-}}{2 \pi}<N\left|A^{+\alpha}\left(y_{1}^{-}\right) A^{+\beta}\left(y_{1}^{-}\right)\right| N>d_{\perp}^{\alpha \beta}
$$

The factors $\eta_{\perp}$ and $\eta_{\|}$are the transverse and longitudinal correlation functions, respectively. $Q^{2} \eta_{\perp}=Q^{2} / R^{2}$ can be schematically explained as the overlap probability of two partons, where $1 / Q$ is the scale of a parton at momentum transfer $Q^{2}$ and $R$ is the maximum correlation length of two partons. Usually, $R$ is regarded as an effective size of nucleon. The nuclear size effect occurs when the scattered quark exceeds the longitudinal size of a nucleon, and it reaches a maximum at $x_{B}=1 /\left(2 R_{A} m_{N}\right)$, where $R_{A}$ is nuclear radius and $m_{N}$ the nucleon mass.

Equation (26) shows a nuclear enhancement mechanism in the higher twist processes, i.e, the rescattering of the outgoing quark on the extra nucleons via the exchange of the contacting gluon-pair can give rise to a visible $A^{1 / 3}$-dependence of the matrix element. We regard a pair of gluons, which are contacted in the $y^{-}$-space as the effective particle $(\Theta$ particle $)$.

From the geometric pictures in Figs. 6 and 7, a straightforward prediction is that the leading contributions to the nuclear size effect in the twist-4 structure functions are from Fig. 6a and its conjugate diagram. However, the oscillating factor can not be suppressed since $y_{1}^{-} \neq y_{2}^{-}$, although a quark pair in Fig. 6c can come from an extra nucleon and forms a colorless cluster. The contributions of Fig. 6b, 6d and 6e are also excluded since there is no contacting gluon pair, which can freely move along the longest path on the 
$y^{-}$-space. Thus, the leading contributions of the geometric effect at twist-4 are only from Figs. 6a and its conjugate diagram, with all the rest diagrams being sub-leading and negligible in the resummation.

The leading diagrams in twist-6 amplitudes are Figs. 10a, 10b and their conjugates, which lead to the nuclear size effect $\sim A^{2 / 3}$. On the other hand, the quark-gluon pairs in Figs. 10c-10e are colored and they are strongly correlated with the other nucleons. Thus, we can not set $x_{1}=x_{2}$ in these diagrams to get the leading nuclear size effect. The integrated path of a contacting gluon-pair, which is connected with other nucleon via a gluon in Figs. 10f, is shorter than that in Fig. 10a. Therefore, we conclude that the contributions of Figs. 10c-10f, 11a-11d (and other diagrams, which have not dawn in Figs. 10 and 11) are $\leq A^{2 / 3}$, and they are excluded in counting the leading effect. In consequence, we need resum only the leading contributions from such CLTOPT diagrams, where a maximum number of contracting gluon-pairs may freely move along $y_{1}^{-}$in a larger nucleus. According to this rule, the contributions of the longitudinal structure functions are excluded in our resummation. Therefore, the geometry of the CLTOPT provides an ideal tool to choose the leading diagrams in the resummation of the geometric nuclear effect. 


\section{Geometric nuclear size effect in structure functions}

We calculate the contributions of higher twist amplitudes to the structure functions in this Section. We begin with the process shown in Fig. 14a. According to the results of the above section, this process provides a leading nuclear effect to the transverse structure function. Using the projection operator and TOPT rules, the contributions of Fig. 14a to the $F_{T}\left(x_{B}, Q^{2}\right)$ at $x_{1} \rightarrow x_{2}$ are

$$
\begin{aligned}
\Delta_{a} F_{T}\left(x_{B}, Q^{2}\right)= & 2 e^{\mu \nu} W^{\mu \nu} \\
= & \sum_{i} 4 \pi e_{i}^{2} \alpha_{s} \int d x_{1} d x_{2}<\frac{1}{2}>_{\text {colour }} d_{\perp}^{\mu \nu} \\
& \times \frac{1}{4} \operatorname{Tr}\left[x_{2} P^{+} \gamma \cdot \bar{n} \gamma^{\mu} \frac{\gamma \cdot n}{2\left(x_{1}-x_{B}\right) P^{+}} \gamma^{\alpha} \gamma \cdot n \frac{x_{B} P^{+}}{Q^{2}} \gamma^{\beta} \frac{Q^{2}}{2 x_{B} P^{+}} \gamma \cdot n \gamma^{\nu}\right] \\
& \times d_{\perp}^{\alpha \beta} \delta\left(x_{2}-x_{B}\right) \frac{x_{B}}{Q^{2}} T_{2 q 2 g} \\
= & \sum_{i} e_{i}^{2} \int d x_{1} d x_{2} \frac{2 \pi \alpha_{s}}{Q^{2} R^{2}} \frac{x_{B} \delta\left(x_{2}-x_{B}\right)}{x_{1}-x_{B}} f_{q_{i}}\left(x_{1}, Q^{2}\right)\left(A^{1 / 3}-1\right) \Theta .
\end{aligned}
$$

Similarly, we have the contributions of Fig. $14 \mathrm{~b}$ to $F_{T}$ at $x_{2} \rightarrow x_{1}$

$$
\Delta_{b} F_{T}\left(x_{B}, Q^{2}\right)=\sum_{i} e_{i}^{2} \int d x_{1} d x_{2} \frac{2 \pi \alpha_{s}}{Q^{2} R^{2}} \frac{x_{B} \delta\left(x_{1}-x_{B}\right)}{x_{2}-x_{B}} f_{q_{i}}\left(x_{1}, Q^{2}\right)\left(A^{1 / 3}-1\right) \Theta
$$

Summing the contributions of Figs. $14 \mathrm{a}$ and $14 \mathrm{~b}$ in the limit $x_{1} \sim x_{2}$ (see Eq. (A.1) in Appendix A) we get

$$
\Delta F_{T}\left(x_{B}, Q^{2}\right)=\sum_{i} e_{i}^{2} \frac{2 \pi \alpha_{s}}{Q^{2} R^{2}}\left(A^{1 / 3}-1\right) \Theta x_{B} \frac{d}{d x_{B}} f_{q_{i}}\left(x_{B}, Q^{2}\right)
$$

Here we point out that the results of hard part using the TOPT coincides with that using the covariant perturbation theory, which was given in [14]. 
We emphasize that the contributions of the geometric nuclear effect to $F_{T}^{\text {twist-4 }}\left(x_{B}, Q^{2}\right)$ are always negative at small $x_{B}$ due to the shape of the quark number density as a function $x_{B}$. Equation (30) means that the double rescattering of an outgoing quark via an effective $\Theta$ particle, which consists of a pair of contacting gluons, reduces the magnitudes of the transverse structure function.

Now we consider the contributions of the higher twist amplitudes and sum over them. We calculate the processes in Fig. 15, where the cut line divides the initial gluons into two parts, each contains an even number of gluons. For simplicity, we indicate the $\Theta$ particle as the dashed line. We have shown that these amplitudes provide maximum nuclear size effects in the structure functions at the twist-6 level. The contributions of Fig. 15a to $F_{T}\left(x_{B}, Q^{2}\right)$ at $x_{1} \rightarrow x_{3}$ is

$$
\Delta_{a} F_{T, t w i s t-6}\left(x_{B}, Q^{2}\right)=\sum_{i} e_{i}^{2} \int d x_{1} d x_{2} d x_{3}\left(\frac{2 \pi \alpha_{s}}{Q^{2} R^{2}}\right)^{2} \frac{x_{B}^{2} \delta\left(x_{3}-x_{B}\right)}{\left(x_{1}-x_{B}\right)\left(x_{2}-x_{B}\right)} 2 x_{3} T_{2 q 4 g}
$$

where the matrix element is

$$
\begin{aligned}
& 2 x_{3} T_{2 q 4 g}\left(x_{1}, Q^{2}\right) \\
= & 2 x_{3} \int \frac{P^{+} d y^{-}}{2 \pi} \frac{P^{+} d y_{1}^{-}}{2 \pi} e^{i x_{1} P^{+} y^{-}} \frac{1}{A} \frac{1}{2 P^{+}} \\
& \times<A\left|\overline{\Psi_{i}}\left(0^{-}\right) \gamma^{+} A^{+\alpha_{1}}\left(y_{1}^{-}\right) A^{+\beta_{1}}\left(y_{1}^{-}\right) \gamma^{+} A^{+\alpha_{2}}\left(y_{2}^{-}\right) A^{+\beta_{2}}\left(y_{2}^{-}\right) \Psi_{i}\left(y^{-}\right)\right| A>d_{\perp}^{\alpha_{1} \beta_{1}} d_{\perp}^{\alpha_{2} \beta_{2}} \\
= & \left(\frac{A^{1 / 3}-1}{R^{2}}\right)^{2} \Theta^{2} f_{q_{i}}\left(x_{B}\right) .
\end{aligned}
$$

Therefore, we obtain

$$
\begin{aligned}
& \Delta_{a} F_{T, \text { twist-6 }}\left(x_{B}, Q^{2}\right) \\
= & \sum_{i} e_{i}^{2} \int d x_{1} d x_{2} d x_{3}\left(\frac{2 \pi \alpha_{s}}{Q^{2} R^{2}}\right)^{2}\left(A^{1 / 3}-1\right)^{2} \Theta^{2} \frac{x_{B}^{2} \delta\left(x_{3}-x_{B}\right)}{\left(x_{1}-x_{B}\right)\left(x_{2}-x_{B}\right)} f_{q_{i}}\left(x_{1}, Q^{2}\right) .
\end{aligned}
$$


Summing the contributions of three cut diagrams in Fig. 15 at the limit $x_{1} \rightarrow x_{3}$, we get (see Eq. (A.2) in Appendix A)

$$
\Delta F_{T, t w i s t-6}\left(x_{B}, Q^{2}\right)=\sum_{i} e_{i}^{2}\left(\frac{2 \pi \alpha_{s}}{Q^{2} R^{2}}\right)^{2}\left(A^{1 / 3}-1\right)^{2} \frac{1}{2 !} \Theta^{2} x_{B}^{2} \frac{d^{2}}{d x_{B}^{2}} f_{q_{i}}\left(x_{B}, Q^{2}\right) .
$$

Similarly, we obtain the contributions from the twist- $(2 n+2)$ amplitudes to $F_{T}\left(x_{B}, Q^{2}\right)$, which contains the exchange of $n-\Theta$ particles (see Eq. (A.3) in Appendix A). The result is

$$
\Delta F_{T, t w i s t-(2 n+2)}\left(x_{B}, Q^{2}\right)=\sum_{i} e_{i}^{2}\left(\frac{2 \pi \alpha_{s}}{Q^{2} R^{2}}\right)^{n}\left(A^{1 / 3}-1\right)^{n} \frac{1}{n !} \Theta^{n} x_{B}^{n} \frac{d^{n}}{d x_{B}^{n}} f_{q_{i}}\left(x_{B}, Q^{2}\right) .
$$

Thus, we can easily write the sum of the contributions from all higher twist amplitudes, which give a leading nuclear size dependence of the transverse structure functions $F_{T}\left(x_{B}, Q^{2}\right)$

$$
\begin{aligned}
\Delta F_{T}\left(x_{B}, Q^{2}\right) & =\sum_{i} e_{i}^{2} \sum_{n=1}^{N}\left(\frac{2 \pi \alpha_{s}}{Q^{2} R^{2}}\right)^{n}\left(A^{1 / 3}-1\right)^{n} \frac{1}{n !} \Theta^{n} x_{B}^{n} \frac{d^{n}}{d x_{B}^{n}} f_{q_{i}}\left(x_{B}, Q^{2}\right) \\
& =\sum_{i} e_{i}^{2}\left[\exp \left(\frac{2 \pi \alpha_{s}}{Q^{2} R^{2}}\left(A^{1 / 3}-1\right) \Theta x_{B} \frac{d}{d x_{B}}\right)-1\right] f_{q_{i}}\left(x_{B}, Q^{2}\right) .
\end{aligned}
$$




\section{Geometric nuclear size effect in evolution equation}

In this section we discuss the contributions of the geometric higher twist effect to the DGLAP equation. We still work in the collinear infinite frame (see Eq. (2)). The coefficient functions with one quark loop corrections and at $L L A\left(Q^{2}\right)$ can be modelled using either the standard scattering picture (Fig. 11) at $p^{+} \gg q^{-}$or the dipole picture (Fig. 16) at $q^{-} \gg p^{+}$(we call the latter frame as the dipole frame). There are different versions of the dipole model. A general dipole model for the DIS [15] is constructed in the $k_{T}$-factorization scheme, where the transverse momenta of the initial partons do not vanish and evolution dynamics is the BFKL equation [16]. In this work we shall show that the dipole picture can also be used in the collinear factorization scheme.

As we have pointed out that the geometric nuclear effect is exposed in the collinear part of the outgoing quark interacting with the nuclear gluons, where the transverse momenta of the partons are negligible. Therefore, the geometric nuclear effect is hidden under the scattering picture Fig. 13, since the contributions of the transverse momentum flowing into the quark-gluon vertex in this picture is necessary in the derivation of the evolution (splitting) kernel.

Different from the scattering picture, in the dipole picture (Fig. 16) the interacting part of quark with gluon (the dashed box in Fig. 16) can be separated from the splitting kernel using TOPT and then we may take the collinear approximation. At first step, we derive the DGLAP equation in the dipole model. The momenta of the partons in Fig. 16 are parameterized as 


$$
q=q^{-} n-\frac{Q^{2}}{2 q^{-}} \bar{n}
$$

i.e.,

$$
\begin{aligned}
& E_{q}=\frac{1}{\sqrt{2}}\left(q^{+}+q^{-}\right) \simeq \sqrt{2} q^{-} \equiv q^{-} \\
& q_{z}=\frac{1}{\sqrt{2}}\left(q^{+}-q^{-}\right) \simeq-\sqrt{2} q^{-}=-q^{\prime-} .
\end{aligned}
$$

Thus, we have

$$
\begin{aligned}
\hat{k}_{D} & \simeq\left(z_{D} q^{\prime-}+\frac{k_{\perp}^{2}}{2 z_{D} q^{-}}, k_{\perp},-z_{D} q^{\prime-}\right) \\
k_{2} & \simeq\left(\left(1-z_{D}\right) q^{\prime-}+\frac{k_{\perp}^{2}}{2\left(1-z_{D}\right) q^{\prime-}},-k_{\perp},-\left(1-z_{D}\right) q^{\prime-}\right) .
\end{aligned}
$$

On the other hand,

$$
p=\left(E_{p}, p_{\perp}, p_{3}\right)=\left(y_{D} P^{+}, 0, y_{D} P^{+}\right)
$$

The relevant coefficient function which contains the splitting function at $L L A\left(Q^{2}\right)$ in the dipole picture is

$$
\begin{aligned}
C_{D}^{\text {twist-2 }} & =\int \frac{d^{3} \vec{k}_{1}}{(2 \pi)^{3}} \frac{1}{8 E_{\hat{k}_{D}} E_{k_{2}} E_{q}}\left[\frac{1}{E_{\hat{k}_{D}}-E_{k_{2}}-E_{q}}\right]^{2} \frac{1}{2} \sum\left|\bar{M}^{D}(\gamma \rightarrow q \bar{q})\right|^{2} \\
& \times \frac{E_{q}}{E_{\hat{k}_{D}}} \frac{1}{4} \sum \bar{M}^{D}(q g \rightarrow q) \frac{1}{z_{D} Q^{2}} \delta\left(z_{D}-\frac{y_{D}}{x_{B}}\right) \\
& \equiv \frac{\sum e_{i}^{2}}{8 \pi^{2}} \int \frac{d k_{\perp}^{2}}{k_{\perp}^{2}} \frac{d y_{D}}{y_{D}} \frac{1}{z_{D} Q^{2}}\left[z_{D}^{2}+\left(1-z_{D}\right)^{2}\right] H^{t w i s t-2},
\end{aligned}
$$

where we introduce the probe function of the gluon 


$$
\begin{aligned}
H^{t w i s t-2} & =\frac{1}{4} g^{2} \operatorname{Tr}\left[z_{D} \frac{Q^{2}}{2 q^{\prime-}} \gamma \cdot \bar{n} \gamma_{\alpha} z_{D} q^{\prime-} \gamma \cdot n \gamma_{\beta}\right] d_{\perp}^{\alpha \beta} \\
& =g^{2} z_{D}^{2} Q^{2}
\end{aligned}
$$

and used the on-shell condition

$$
\delta\left(\left(p+z_{D} q\right)^{2}\right)=\frac{1}{z_{D} Q^{2}} \delta\left(z_{D}-\frac{y_{D}}{x_{B}}\right)
$$

In the calculations of $\sum\left|\bar{M}^{D}(\gamma \rightarrow q \bar{q})\right|^{2}$, we regard the virtual photon as an equivalent photon in the Weizsäcker-Williams approximation [17] under condition $q^{-} \gg p^{+}$. In this case, we consider the contributions of the transverse polarized photon and define $z_{D}$. Note that the longitudinal coefficient function doesn't contribute to the DGLAP dynamics.

The splitting function is defined as

$$
\frac{C_{D}^{t w i s t-2}}{2 z_{D} \sum e_{i}^{2}}=\frac{\alpha_{s}}{2 \pi} \int \frac{d k_{\perp}^{2}}{k_{\perp}^{2}} \frac{d y_{D}}{y_{D}} P_{q g}^{D}\left(z_{D}\right)
$$

i.e.,

$$
P_{q g}^{D}\left(z_{D}\right)=\frac{1}{2}\left[z_{D}^{2}+\left(1-z_{D}\right)^{2}\right]
$$

Using the factorized splitting function, we can construct a DGLAP equation in the dipole picture (see Appendix B and [18])

$$
\frac{d f_{q_{i}}\left(x_{B}, Q^{2}\right)}{d \ln Q^{2}}=\frac{\alpha_{s}}{2 \pi} \int_{x_{B}^{2}}^{x_{B}} \frac{d y_{D}}{y_{D}} P_{q_{i} g}^{D}\left(\frac{y_{D}}{x_{B}}\right) f_{g}\left(y_{D}, Q^{2}\right)
$$

where $x_{B}^{2} \leq y_{D} \leq x_{B}$. 
Comparing Eq. (46) with the DGLAP equation in the scattering picture, we have the following relations between the scattering and dipole pictures due to the different definitions about $z_{D}$ and $z_{S}$ :

$$
P_{q g}^{S}\left(z_{S}\right) \leftrightarrow P_{q g}^{D}\left(z_{D}\right)
$$

where $z_{D}=y_{D} / x_{B}, z_{S}=x_{B} / y_{S}$ and we use "D" and "S" to indicate the quantities in the dipole and scattering pictures, respectively.

We emphasize that although both $y_{D}$ and $y_{S}$ are defined as the momentum fractions of the target carried by the gluon, they have different kinematical regions. It is interesting to note that a smaller value of $x_{B}$ corresponds to a smaller value of $y_{D}$ in the dipole model since $x_{B}=y_{D} / z_{D}$; on the other hand, in the scattering picture the gluons can take a larger momentum fraction $y_{S}$ of proton-momentum even in the small $x_{B}$ region since $z_{S}=x_{B} / y_{S} \leq 1$

Using Eq. (47), one can rewrite Eq. (46) as a standard DGLAP equation

$$
\frac{d f_{q_{i}}\left(x_{B}, Q^{2}\right)}{d \ln Q^{2}}=\frac{\alpha_{s}}{2 \pi} \int_{x_{B}}^{1} \frac{d y_{S}}{y_{S}} P_{q g}^{S}\left(\frac{x_{B}}{y_{S}}\right) f_{g}\left(y_{S}, Q^{2}\right)
$$

where $x_{B} \leq y_{S} \leq 1$

In our dipole model the splitting kernel containing loop transverse momentum $k_{\perp}$ is separated from the probe function $H^{\text {twist-2 }}$, in which we can take the collinear approximation, i.e., neglecting the contributions of the loop transverse momentum.

Now we discuss the corrections of the geometric nuclear effect to the DGLAP equation using the dipole picture. The nuclear gluons can collinearly attach to two quark legs in 
any ways. However, the leading contributions to the geometric nuclear effect are from following probe functions:

$$
H^{\text {twist-4 }}=H^{t w i s t-2} 2 \pi \alpha_{s}\left(A^{1 / 3}-1\right) \frac{x_{B}^{3}}{y_{D} Q^{2} R^{2}} \Theta \frac{d}{d y_{D}} f_{g}\left(y_{D}, Q^{2}\right)
$$

at twist-4 (see Fig. 17), and

$$
H^{t w i s t-6}=H^{t w i s t-2}\left(2 \pi \alpha_{s}\right)^{2}\left(A^{1 / 3}-1\right)^{2} \frac{x_{B}^{5}}{y_{D}^{2} Q^{4} R^{4}} \Theta^{2} \frac{1}{2 !} \frac{d^{2}}{d y_{D}^{2}} f_{g}\left(y_{D}, Q^{2}\right) .
$$

at twist-6 (see Fig. 18).

We sum the leading contributions of the nuclear size-effect at all order of twist to the DGLAP equation, the result is

$$
\begin{aligned}
& \frac{d f_{q_{i}}\left(x_{B}, Q^{2}\right)}{d \ln Q^{2}} \\
& =\frac{\alpha_{s}}{2 \pi} \int_{x_{B}}^{1} \frac{d y_{S}}{y_{S}} P_{q_{i} g}^{S}\left(\frac{x_{B}}{y_{S}}\right) f_{g}\left(y_{S}, Q^{2}\right) \\
& +\frac{\alpha_{s}}{2 \pi} \int_{x_{B}^{2}}^{x_{B}} \frac{d y_{D}}{y_{D}} P_{q_{i} g}^{D}\left(\frac{y_{D}}{x_{B}}\right)\left\{x_{B}\left[\exp \left(2 \pi \alpha_{s} \frac{x_{B}^{2}}{y_{D} Q^{2} R^{2}}\left(A^{1 / 3}-1\right) \Theta \frac{d}{d y_{D}^{\prime}}\right)-1\right] f_{g}\left(y_{D}^{\prime}, Q^{2}\right)\right\}_{y_{D}^{\prime}=y_{D}} .
\end{aligned}
$$

We don't need to consider the nuclear size-effect on the gluon legs since these diagrams are excluded as the sub-leading contributions in our resummation. 


\section{Discussions and summary}

One of our results, Eq. (36), is consistent with the Qiu-Vitev resummation for $\Delta F_{T}\left(x_{B}, Q^{2}\right)$ in Ref. [6], only differing from an unknown constant. The reason is that these two approaches are based on different dynamics. The derivation of the Qiu-Vitev formula assumes that the leading contributions to the nuclear size effect in the structure functions are arisen from the singularities of the gluon correlation functions. This argument does not indicate a lot of neglecting graphs, which give the sub-leading contributions. In this respect, the geometry of the CLTOPT improves the Qiu-Vitev derivation.

It is interesting to compare Eq. (51) with the Mueller-Glauber model [2]. These two models are written in the dipole picture but using different factorization schemes. Besides, the Glauber approach of the multiple scattering theory is based on the eikonal approximation, which assumes that the scattering on different nucleons are incoherent. Thus, the phase shifts from each scattering can sum up to an exponential form. In the TOPT-language, the eikonal approximation implies that all propagators connecting with nuclear gluons take their forward component. On the other hand, Eq. (51) provides a different picture, where the backward propagator alternates with the forward one when scattered quark passes through the nucleus.

It is well known that there is an obvious nuclear screening effect in the structure function $F_{2}^{A}$ at small $x_{B}$ [19]. As we have pointed out that the nuclear size effect in the longitudinal stricture function at leading order vanishes. The nuclear screening effect at small $x_{B}$ region can be expressed in the ratios of the structure functions as

$$
R\left(x_{B}, Q^{2}\right)=\frac{F_{A, 2}\left(x_{B}, Q^{2}\right)}{F_{N, 2}\left(x_{B}, Q^{2}\right)}
$$




$$
\begin{aligned}
& =\frac{x_{B} F_{A, L}\left(x_{B}, Q^{2}\right)+x_{B} F_{A, T}\left(x_{B}, Q^{2}\right.}{F_{N, 2}\left(x_{B}, Q^{2}\right)} \\
& \simeq 1+\frac{x_{B} \Delta F_{T}\left(x_{B}, Q^{2}\right)}{F_{N, 2}\left(x_{B}, Q^{2}\right)} .
\end{aligned}
$$

From these results, one can fix the parameter $\Theta$.

The nuclear PDFs are one of fundamental knowledge for understanding the experimental data at the Relativistic Heavy Ion Collider (RHIC) and Large Hadron Collider (LHC). The PDFs are calculable if the nonperturbative input distributions and their QCD evolution dynamics are known. Therefore, the corrections of nuclear circumstances to the evolution equations and input distributions become an active topic. The nuclear PDFs can be modelled using the DGLAP equation from the relating DIS data [20]. The results show that the input PDFs in the bound nucleon differ by the shadowing factors from that in the free nucleon. However, this result does not means that the corrections from the higher twist effects in such researches are unimportant since the shadowing factors are phenomenological. In fact, the nonlinear modification of the gluon recombination to the DGLAP equation as a higher twist effect can't be neglected even in DIS on the free nucleon [21]. As mentioned already in works [6], the higher twist resummation may be of importance in the extraction of the nuclear input PDFs. Our results remind us of that, except the contributions of the gluon recombination effect to the DGLAP equation, the geometric higher twist effect in the nuclear input PDFs and QCD evolution equation should be considered in extracting the nuclear parton distributions from the measured DIS cross sections.

As we have mentioned that the higher twist effects and their summation may provide a valuable information for the multi-parton correlations at long distance in the target. The geometric nuclear effect presents an interesting freedom in high energy nuclear physics- 
the contacting gluon-pair, which is soft at the hadronic level since it carries almost zeromomentum $\left(x_{1}-x_{2} \simeq 0\right)$, however, the effect is really calculable in the perturbative QCD.

In summary, the higher twist effect in deep inelastic scattering on the nuclear target are studied using time ordered perturbation theory. We have shown that the collinear rescattering of the outgoing quark on the extra nucleon via the contacting gluon-pair dominates a nuclear size dependent effect. The leading contributions of nuclear-enhanced effect to the structure function and DGLAP evolution equation are resummed by using the geometric properties of the higher twist amplitudes.

Acknowledgments: This work was supported by National Natural Science Foundations of China 10075020, 50193013, and 10475028. 


\section{Appendix A:}

We list some algebraic identities in the derivations in Section 5.

In $F_{T, t w i s t-4}$

$$
\begin{aligned}
& \int d x_{1} d x_{2}\left[\frac{\delta\left(x_{2}-x_{B}\right)}{x_{1}-x_{B}}+\frac{\delta\left(x_{1}-x_{B}\right)}{x_{2}-x_{B}}\right] \delta\left(x_{2}-x_{1}-\Delta\right) \\
& =-\int d x_{1}\left[\frac{\delta\left(x_{1}-x_{B}+\Delta\right)}{\Delta}-\frac{\delta\left(x_{1}-x_{B}\right)}{\Delta}\right] \\
& =-\int d x_{1}\left[\delta^{\prime}\left(x_{1}-x_{B}\right)\right] \\
& =\frac{d}{d x_{B}},
\end{aligned}
$$

when $\Delta \rightarrow 0$.

In $F_{T, t w i s t-6}$

$$
\begin{aligned}
& \int d x_{1} d x_{2} d x_{3}\left[\frac{\delta\left(x_{3}-x_{B}\right)}{\left(x_{1}-x_{B}\right)\left(x_{2}-x_{B}\right)}+\frac{\delta\left(x_{2}-x_{B}\right)}{\left(x_{1}-x_{B}\right)\left(x_{3}-x_{B}\right)}+\frac{\delta\left(x_{1}-x_{B}\right)}{\left(x_{2}-x_{B}\right)\left(x_{3}-x_{B}\right)}\right] \\
& \times \delta\left(x_{2}-x_{1}-\Delta\right) \delta\left(x_{3}-x_{2}-\Delta\right) \\
& =\int d x_{1} d x_{2} d x_{3}\left[\frac{\delta\left(x_{3}-x_{B}\right)}{2 \Delta^{2}}-\frac{\delta\left(x_{2}-x_{B}\right)}{\Delta^{2}}+\frac{\delta\left(x_{1}-x_{B}\right)}{2 \Delta^{2}}\right] \delta\left(x_{2}-x_{1}-\Delta\right) \delta\left(x_{3}-x_{2}-\Delta\right) \\
& =-\int d x_{1} d x_{2} \frac{1}{2 \Delta}\left[\delta^{\prime}\left(x_{1}-x_{B}\right)-\delta^{\prime}\left(x_{2}-x_{B}\right)\right] \delta\left(x_{2}-x_{1}-\Delta\right) \\
& =-\int d x_{1} \frac{1}{2 \Delta}\left[\delta^{\prime}\left(x_{1}-x_{B}\right)-\delta^{\prime}\left(x_{1}-x_{B}+\Delta\right)\right] \\
& =\int d x_{1} \frac{1}{2} \delta^{\prime \prime}\left(x_{1}-x_{B}\right) \\
& =\frac{1}{2 !} \frac{d^{2}}{d x_{B}^{2}}
\end{aligned}
$$

when $\Delta \rightarrow 0$.

In $F_{T, t w i s t-8}$ 


$$
\begin{aligned}
& \int d x_{1} d x_{2} d x_{3} d x_{4}\left[\frac{\delta\left(x_{4}-x_{B}\right)}{\left(x_{1}-x_{B}\right)\left(x_{2}-x_{B}\right)\left(x_{3}-x_{B}\right)}+\frac{\delta\left(x_{3}-x_{B}\right)}{\left(x_{1}-x_{B}\right)\left(x_{2}-x_{B}\right)\left(x_{4}-x_{B}\right)}\right. \\
& \left.+\frac{\delta\left(x_{2}-x_{B}\right)}{\left(x_{1}-x_{B}\right)\left(x_{3}-x_{B}\right)\left(x_{4}-x_{B}\right)}+\frac{\delta\left(x_{B}\right)}{\left(x_{2}-x_{B}\right)\left(x_{3}-x_{B}\right)\left(x_{4}-x_{B}\right)}\right] \\
& \times \delta\left(x_{2}-x_{1}-\Delta\right) \delta\left(x_{3}-x_{2}-\Delta\right) \delta\left(x_{4}-x_{3}-\Delta\right) \\
& =\int d x_{1} d x_{2} d x_{3} d x_{4}\left[-\frac{\delta\left(x_{4}-x_{B}\right)}{6 \Delta^{3}}+\frac{\delta\left(x_{3}-x_{B}\right)}{2 \Delta^{3}}-\frac{\delta\left(x_{2}-x_{B}\right)}{2 \Delta^{3}}+\frac{\delta\left(x_{1}-x_{B}\right)}{6 \Delta^{3}}\right] \\
& \times \delta\left(x_{2}-x_{1}-\Delta\right) \delta\left(x_{3}-x_{2}-\Delta\right) \delta\left(x_{4}-x_{3}-\Delta\right) \\
& =-\int d x_{1} d x_{2} d x_{3} \frac{1}{6 \Delta^{2}}\left[\delta^{\prime}\left(x_{3}-x_{B}\right)-\delta^{\prime}\left(x_{2}-x_{B}\right)-\delta^{\prime}\left(x_{2}-x_{B}\right)+\delta^{\prime}\left(x_{1}-x_{B}\right)\right] \\
& \times \delta\left(x_{2}-x_{1}-\Delta\right) \delta\left(x_{3}-x_{2}-\Delta\right) \\
& =-\int d x_{1} \frac{1}{6 \Delta}\left[\delta^{\prime \prime}\left(x_{1}-x_{B}+\Delta\right)-\delta^{\prime \prime}\left(x_{1}-x_{B}\right)\right] \\
& =-\int d x_{1} \frac{1}{6} \delta^{\prime \prime \prime}\left(x_{1}-x_{B}\right) \\
& =\frac{1}{3 !} \frac{d^{3}}{d x_{B}^{3}},
\end{aligned}
$$

when $\Delta \rightarrow 0$. 


\section{Appendix B:}

In this Appendix we derive a set of complete DGLAP evolution equations in the dipole frame. At first step, we give the definitions of the parton distributions in this frame. The QCD evolution equations of the PDFs are irrelevant to the probe, therefor, we may use a virtual gluon or a virtual quark, which connects with an extra color source "S", to replace the virtual photon in DIS. Thus, the first order of the DIS amplitudes in the dipole frame, can be expressed as the four graphs shown in Fig. 19.

We take Fig. 19a as an example. The cross section of a virtual gluon scattering off a proton in the dipole frame and in the collinear factorization scheme is

$$
d \sigma_{a}\left(q P \rightarrow k_{1} X\right)=\int d y f_{g}(y) d \sigma_{a}\left(q p \rightarrow k_{1}\right) .
$$

For convenience, we neglect the symbol "D" for the dipole picture. According to the parton model,

$$
d \sigma_{a}\left(q p \rightarrow k_{1}\right)=C_{a} \delta\left(y-x_{B}\right),
$$

where $C_{a}$ is a function describing the probe vertex. In the above equation and below, we will use the momenta of the partons to represent the particles entering cross sections and matrices. The gluon distribution in the proton at the first order QCD can now be defined as

$$
f_{g}\left(x_{B}\right)=\frac{1}{C_{a}} d \sigma_{a}\left(q P \rightarrow k_{1} X\right) .
$$

Similarly, we have three more definitions corresponding to Fig. 19b-19d. 


$$
\begin{aligned}
& f_{g}\left(x_{B}\right)=\frac{1}{C_{b}} d \sigma_{b}\left(q P \rightarrow k_{1} X\right), \\
& f_{q}\left(x_{B}\right)=\frac{1}{C_{c}} d \sigma_{c}\left(q P \rightarrow k_{1} X\right),
\end{aligned}
$$

and

$$
f_{q}\left(x_{B}\right)=\frac{1}{C_{d}} d \sigma_{d}\left(q P \rightarrow k_{1} X\right)
$$

On the other hand, we can write the formulae for the four cross sections of Fig. 19 in the TOPT form as

$$
\begin{gathered}
d \sigma_{a, b}\left(q P \rightarrow k_{1} X\right) \\
=\frac{E_{p}}{E_{P}}\left|\bar{M}_{g}(P \rightarrow p X)\right|^{2}\left[\frac{1}{E_{P}-E_{p}-E_{X}}\right]^{2}\left[\frac{1}{2 E_{p}}\right]^{2} \prod_{X} \frac{d^{3} k_{X}}{(2 \pi)^{3} 2 E_{X}} \\
\times \frac{1}{8 E_{p} E_{q}} \bar{M}_{a, b}\left(p \rightarrow q k_{1} \rightarrow p\right)(2 \pi)^{4} \delta^{4}\left(p-q-k_{1}\right) \frac{d^{3} k_{1}}{(2 \pi)^{3} 2 E_{k_{1}}},
\end{gathered}
$$

and

$$
\begin{gathered}
d \sigma_{c, d}\left(q P \rightarrow k_{1} X\right) \\
=\frac{E_{p}}{E_{P}}\left|\bar{M}_{q}(P \rightarrow p X)\right|^{2}\left[\frac{1}{E_{P}-E_{p}-E_{X}}\right]^{2}\left[\frac{1}{2 E_{p}}\right]^{2} \prod_{X} \frac{d^{3} k_{X}}{(2 \pi)^{3} 2 E_{X}} \\
\times \frac{1}{8 E_{p} E_{q}} \bar{M}_{c, d}\left(p \rightarrow q k_{1} \rightarrow p\right)(2 \pi)^{4} \delta^{4}\left(p-q-k_{1}\right) \frac{d^{3} k_{1}}{(2 \pi)^{3} 2 E_{k_{1}}} .
\end{gathered}
$$

Comparing Eqs. (B.7) and (B.8) with (B.1), we get the definitions of the quark and gluon distributions 


$$
\begin{aligned}
& f_{g}(y) d y=\frac{E_{p}}{E_{P}}\left|\bar{M}_{g}(P \rightarrow p X)\right|^{2}\left[\frac{1}{E_{P}-E_{p}-E_{X}}\right]^{2}\left[\frac{1}{2 E_{p}}\right]^{2} \prod_{X} \frac{d^{3} k_{X}}{(2 \pi)^{3} 2 E_{X}}, \\
& f_{q}(y) d y=\frac{E_{p}}{E_{P}}\left|\bar{M}_{q}(P \rightarrow p X)\right|^{2}\left[\frac{1}{E_{P}-E_{p}-E_{X}}\right]^{2}\left[\frac{1}{2 E_{p}}\right]^{2} \prod_{X} \frac{d^{3} k_{X}}{(2 \pi)^{3} 2 E_{X}},
\end{aligned}
$$

and the probe vertices

$$
\begin{gathered}
\frac{1}{C_{j}} d \sigma_{j}\left(q p \rightarrow k_{1}\right) \\
=\frac{1}{C_{j}} \frac{1}{8 E_{p} E_{q}} \bar{M}_{j}\left(p \rightarrow q k_{1} \rightarrow p\right)(2 \pi)^{4} \delta^{4}\left(p-q-k_{1}\right) \frac{d^{3} k_{1}}{(2 \pi)^{3} 2 E_{k_{1}}} \\
=\delta\left(y-x_{B}\right)
\end{gathered}
$$

in the TOPT form, where $j=a, b, c$ and $d$.

When the scale $Q^{2}$ is increased by a small amount due to the radiation of a parton, i.e., $Q^{2} \rightarrow Q^{2}+\Delta Q^{2}$, the corresponding change of the PDFs, for example in Fig. 20a' is

$$
\begin{gathered}
d f_{g}\left(x_{B}\right) \\
=\frac{1}{C_{a}} d \sigma_{a^{\prime}}\left(q P \rightarrow k_{1} k_{2} X\right) \\
=\frac{E_{p}}{E_{P}}\left|\bar{M}_{g}(P \rightarrow p X)\right|^{2}\left[\frac{1}{E_{P}-E_{p}-E_{X}}\right]^{2}\left[\frac{1}{2 E_{p}}\right]^{2} \prod_{X} \frac{d^{3} k_{X}}{(2 \pi)^{3} 2 E_{X}} H_{a}(q p \rightarrow q p) \\
=\int d y f_{q}(y) H_{a}(q p \rightarrow q p),
\end{gathered}
$$

where

$$
H_{a}(q p \rightarrow q p)
$$




$$
\begin{gathered}
=\frac{1}{C_{a}} d \sigma_{a}\left(p \rightarrow z_{D} q k_{1} \rightarrow p\right) \\
\frac{E_{\hat{k}}}{E_{q}}\left|\bar{M}\left(q \rightarrow \hat{k} k_{2}\right)\right|^{2}\left[\frac{1}{E_{\hat{k}}-E_{k_{2}}-E_{q}}\right]^{2}\left[\frac{1}{2 E_{\hat{k}}}\right]^{2} \frac{d^{3} k_{2}}{(2 \pi)^{3} 2 E_{k_{2}}} \\
=\frac{1}{y} \delta\left(z_{D}-\frac{y}{x_{B}}\right) \frac{d k_{\perp}^{2}}{k_{\perp}^{2}} \frac{\alpha_{s}}{2 \pi} P_{g g}\left(z_{D}\right),
\end{gathered}
$$

where $P_{g g}\left(z_{D}\right)$ is the splitting function for $g \rightarrow g g$. The momenta of the partons in Fig. 20 are parameterized as Eqs. (39) and (40). From Eqs. (B.12) and (B.13), we get a DGLAP evolution equation

$$
\frac{d f_{g}\left(x_{B}, Q^{2}\right)}{d \ln Q^{2}}=\frac{\alpha_{s}}{2 \pi} \int_{x_{B}^{2}}^{x_{B}} \frac{d y}{y} f_{g}\left(y, Q^{2}\right) P_{g g}\left(\frac{y}{x_{B}}\right),
$$

and

$$
P_{g g}\left(z_{D}\right)=2 C_{2}(G)\left[\frac{1-z_{D}}{z_{D}}+\frac{z_{D}}{1-z_{D}}+z_{D}\left(1-z_{D}\right)\right]
$$

Along similar lines, we derived the evolution equations corresponding to Figs. 20b'20d'

$$
\begin{gathered}
\frac{d f_{q_{i}}\left(x_{B}, Q^{2}\right)}{d \ln Q^{2}}=\frac{\alpha_{s}}{2 \pi} \int_{x_{B}^{2}}^{x_{B}} \frac{d y}{y} f_{g}\left(y, Q^{2}\right) P_{q_{i} g}\left(\frac{y}{x_{B}}\right), \\
\frac{d f_{g}\left(x_{B}, Q^{2}\right)}{d \ln Q^{2}}=\frac{\alpha_{s}}{2 \pi} \sum_{i=1}^{2 f} \int_{x_{B}^{2}}^{x_{B}} \frac{d y}{y} f_{q_{i}}\left(y, Q^{2}\right) P_{g q_{i}}\left(\frac{y}{x_{B}}\right),
\end{gathered}
$$

and

$$
\frac{d f_{q_{i}}\left(x_{B}, Q^{2}\right)}{d \ln Q^{2}}=\frac{\alpha_{s}}{2 \pi} \int_{x_{B}^{2}}^{x_{B}} \frac{d y}{y} f_{q_{j}}\left(y, Q^{2}\right) P_{q_{i} q_{j}}\left(\frac{y}{x_{B}}\right) .
$$

The splitting functions are

$$
P_{q_{i} g}\left(z_{D}\right)=\frac{1}{2}\left[z_{D}^{2}+\left(1-z_{D}\right)^{2}\right]
$$




$$
P_{g q_{i}}\left(z_{D}\right)=C_{2}(R)\left[\frac{1+\left(1-z_{D}\right)^{2}}{z_{D}}\right]
$$

and

$$
P_{q_{i} q_{j}}\left(z_{D}\right)=C_{2}(R)\left[\frac{1+z_{D}^{2}}{1-z_{D}}\right] .
$$

Note that two partons indicated $\hat{k}$ in Fig. 20 are on mass-shell in the TOPT framework, therefore, one can extract the probe function $H^{\text {twist-2 }}$ from the amplitude (Fig. 20b') and generalize it to the higher twist processes as in Section 6.

The probabilistic form of the splitting functions in Eqs. (B.15) and (B.21) have 1/(1$\left.z_{D}\right)$ singularities, which are caused by the emission of a low-momentum gluon. Physically, the infrared singularities can be cancelled when the virtual diagrams, for example Fig. 21 are added to the real contributions. As we have pointed out in Ref. [22], an advantage of TOPT is that it exposes the relation between the real and virtual diagrams without an actual calculation of the virtual diagrams. In fact, from the TOPT-decomposition of the diagrams, we can easily get

$$
\begin{gathered}
\frac{\alpha_{s}}{2 \pi} \frac{d k_{T}^{2}}{k_{T}^{2}} P_{g g}^{v i r t u a l} \\
=\frac{1}{2} \frac{1}{8 E_{\hat{k}} E_{k_{2}}} \frac{1}{E_{\hat{k}}+E_{k_{2}}-E_{q}} \frac{1}{E_{q}-E_{\hat{k}}-E_{k_{2}}}\left|\bar{M}\left(g \rightarrow \hat{k} k_{2}\right)\right|^{2} \frac{d^{3} \vec{k}_{2}}{(2 \pi)^{3}} \\
=-\frac{1}{2} \frac{\alpha_{s}}{2 \pi} \frac{d k_{T}^{2}}{k_{T}^{2}} P_{g g},
\end{gathered}
$$

where $d^{3} \vec{k}_{2}$ is the loop-momentum integral; the factor $1 / 2$ can be explained as follows: only half of the probe-vertex connects with the partonic matrix in the virtual diagrams. That is 


$$
\frac{\sqrt{f_{g}} d \sqrt{f_{g}}}{d \ln Q^{2}}=\frac{1}{2} \frac{d f_{g}}{d \ln Q^{2}} .
$$

The contributions from others virtual diagrams can be derived by similar method. In consequence, we get the evolution equations

$$
\begin{gathered}
\frac{d f_{q_{i}}\left(x_{B}, Q^{2}\right)}{d \ln Q^{2}} \\
=\frac{\alpha_{s}}{2 \pi} \int_{x_{B}^{2}}^{x_{B}} \frac{d y}{y} f_{q_{j}}\left(y, Q^{2}\right) P_{q_{i} q_{j}}\left(\frac{y}{x_{B}}\right)-\frac{\alpha_{s}}{2 \pi} f_{q_{j}}\left(x_{B}, Q^{2}\right) \int_{x_{B}}^{1} d z_{D} P_{q_{i} q_{j}}\left(z_{D}\right) \\
+\frac{\alpha_{s}}{2 \pi} \int_{x_{B}^{2}}^{x_{B}} \frac{d y}{y} f_{g}\left(y, Q^{2}\right) P_{q_{i} g}\left(\frac{y}{x_{B}}\right),
\end{gathered}
$$

and

$$
\begin{gathered}
\frac{d f_{g}\left(x_{B}, Q^{2}\right)}{d \ln Q^{2}} \\
=\frac{\alpha_{s}}{2 \pi} \int_{x_{B}^{2}}^{x_{B}} \frac{d y}{y} \sum_{i=1}^{2 f} f_{q_{i}}\left(y, Q^{2}\right) P_{g q_{i}}\left(\frac{y}{x_{B}}\right)-\frac{\alpha_{s}}{2 \pi} \sum_{i=1}^{2 f} f_{q_{i}}\left(x_{B}, Q^{2}\right) \int_{x_{B}}^{1} d z_{D} P_{g q_{i}}\left(z_{D}\right) \\
+\frac{\alpha_{s}}{2 \pi} \int_{x_{B}^{2}}^{x_{B}} \frac{d y}{y} f_{g}\left(y, Q^{2}\right) P_{g g}\left(\frac{y}{x_{B}}\right)-\frac{\alpha_{s}}{2 \pi} f_{g}\left(x_{B}, Q^{2}\right) \int_{x_{B}}^{1} d z_{D} P_{g g}\left(z_{D}\right) .
\end{gathered}
$$




\section{References}

[1] G. Sterman, An Introduction to Quantum Field Theory (Cambridge Univ. Press, Cambridge, 1993).

[2] A.H. Mueller, Nucl. Phys. B 335 (1990) 115, B 415 (1994) 375 (1994); B 437 (1995) 107.

[3] J. Jalilian-Marian, A. Kovner, L. McLerran, and H. Weigert, Phys. Rev. D 55, 5414 1997!; J. Jalilian-Marian, A. Kovner, A. Leonidov, and H. Weigert, Nucl. Phys. B504 (1997) 415; Phys. Rev. D 59 (1999) 014014; H. Weigert, Nucl. Phys. A 703 (2002) 823; E. Iancu, A. Leonidiv, and L. McLerran, ibid. A 692 (2001) 583 ; Phys. Lett. B 510 (2001) 133.

[4] A.H. Mueller, Phys. Lett. B 525 (2001) 243.

[5] C. S. Lam, G. Mahlon and W. Zhu, Phys. rev. D 66 (2002) 074005.

[6] J.W. Qiu and I. Vitev, Phys.Lett. B587 (2004) 52; arXiv:hep-ph/0309094 and 0405068.

[7] X.F. Guo, J.W. Qiu and W. Zhu, Phys. Lett. B 523 (2001) 88.

[8] M.D. Scadron, 1979. Advanced Quantum theory and Its Applicatiopns Through Feynman Diagrams, eds W. Beigböck, R.P. Geroch, E.H. Lieb, T. Regge and W. Thirring (Springer-Verlag, Berlin Heidelberg) p. 159.

[9] W. Zhu, H.W. Xiong and J.H. Ruan, Phys. Rev. D60 (1999) 094006.

[10] G. Altarelli and G. Parisi, Nucl. Phys. B126 (1977) 298. 
[11] V.N. Gribov and L.N. Lipatov, Sov. J. Nucl. Phys. 15 (1972) 438; Yu.L. Dokshitzer, Sov. Phys. JETP. 46 (1977) 641

[12] J.C. Collins, D.E. Soper and G. Sterman, 1989, Perturbative Quantum Chromodynamics, ed A.H. Mueller (World Scientific) p. 1.

[13] J.W. Qiu and G. Sterman, Phys. Rev. D 49 (1994) 4493; Phys. Rev. D 50 (1994) 1951.

[14] X.F. Guo and J.W. Qiu, arXiv:hep-ph/9810548.

[15] A. H. Mueller, Parton Saturation-An Overview, hep-ph/0111244, Published in QCD Perspectives on Hot and Dense Matter, Eds. J.-P. Blaizot and E. Iancu, NATO Science Series, Kluwer, (2002) and references therein.

[16] L.N. Lipatov, Sov. J. Nucl. Phys. 23 (1976) 338; V. S. Fadin, E.A. Kuraev and L.N. Lipatov, Phys. Lett. B 60 (1975) 50; E.A. Kuraev, L.N. Lipatov and V. S. Fadin, Sov. Phys. JETP 44 (1976) 443; E.A. Kuraev, L.N. Lipatov and V. S. Fadin, Sov. Phys. JETP 45 (1977) 199; I. I. Balitsky and L.N. Lipatov, Sov. J. Nucl. Phys. 28 (1978) 822; I. I. Balitsky and L.N. Lipatov, JETP Lett. 30 (1979) 355.

[17] von Weizsäcker, C.F., 1934. Z. Phys. 88, 612; Williams, E.J., 1934. Phys. Rev. 45, 729; P. Kessler, Nuovo Comento 16 (1966) 809; V.N Baier, V.S. Fadin and V.A. Khoze, Nucl. Phys. 65 (1973) 381; M.S. Chen and P. Zerwas, Phys. Rev. D12 (1975)187.

[18] Z.Q. Shen and W. Zhu, Chinese Phys. Lett. 21 (2004) 1896.

[19] For a review, see M. Arneodo, Phys. Rep. 240 (1994) 301, and references therein. 
[20] K.J. Eskola, H. Honkanen, V.J. Kolhinen, P.V. Ruuskane and C.A. Salgado, J. Phys. G29 (2003) 1947; M. Hirai, S. Kumano, and T.H. Nagai, Phys.Rev. C70 (2004) 044905.

[21] K.J. Eskola, H. Honkanen, V.J. Kolhinen, Jianwei Qiu, and C.A. Salgado, Nucl. Phys. B660 (2003) 211; W. Zhu, J.H. Ruan, J.F. Yang and Z.Q. Shen, Phys. Rev. D68 (2003) 094015.

[22] W. Zhu, Nucl.Phys. B551 (1999) 245; W. Zhu and J.H. Ruan, Nucl. Phys. B559 (1999) 378; W. Zhu, Z.Q. Shen and J.H. Ruan, Nucl. Phys. B692 (2004) 417. 


\section{Figure Captions}

Fig. 1 Elemental vertices of QCD at the collinear limit.

Fig. 2 Elemental vertices of QED at the collinear limit.

Fig. 3 The twist- 4 TOPT diagrams at lowest order for $F_{T}\left(x_{B}, Q^{2}\right)$, the conjugant diagrams have not drawn.

Fig. 4 As similar to Fig. 3, but for $F_{L}\left(x_{B}, Q^{2}\right)$.

Fig. 5 An example of time-space picture corresponding to Fig. 3a.

Fig. 6 Space-time pictures of the amplitudes corresponding to Fig. 3.

Fig. 7 Space-time pictures of the amplitudes corresponding to Fig. 4.

Fig. 8 A part of twist-6 TOPT diagrams at lowest order for $F_{T}\left(x_{B}, Q^{2}\right)$, the conjugant diagrams have not drawn.

Fig. 9 A part of twist-6 TOPT diagrams at lowest order for $F_{L}\left(x_{B}, Q^{2}\right)$, the conjugant diagrams have not drawn.

Fig. 10 Space-time pictures of the amplitudes corresponding to Fig. 8.

Fig. 11 Space-time pictures of the amplitudes corresponding to Fig. 9.

Fig. 12 The naive parton model, which defines the quark distributions.

Fig. 13 The leading order contribution of gluon to $F_{T}\left(x_{B}, Q^{2}\right)$ in the standard parton (scattering) model.

Fig. 14 The leading perturbative contribution to the nuclear size-dependent of $F_{T}\left(x_{B}, Q^{2}\right)$ at twist-4, where an outgoing quark rescattering on the extra nucleon via the contacting colorless gluon-pair exchange.

Fig. 15 The leading diagrams at the twist-6 level, where using the dashed line to denote a contacting colorless gluon pair. 
Fig. 16 Schematic diagrams for the decompositions of a DGLAP equation at the dipole frame in TOPT.

Fig. 17 Space-time picture for the probing function at twist-4, which gives the leading contribution to the nuclear size-dependent of the DGLAP equation.

Fig. 18 Similar to Fig. 17, but at twist-6.

Fig. 19 The definitions of the parton distributions in the dipole frame. Where $S$ is the color source.

Fig. 20 The one-loop corrections to the parton distributions in the dipole picture.

Fig. 21 The virtual diagrams corresponding to Fig. 20a. 


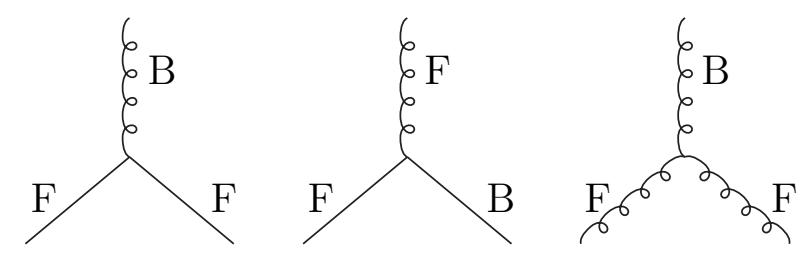

Fig.1 

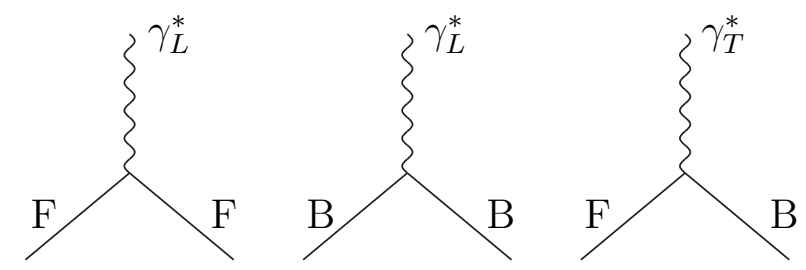

Fig.2 


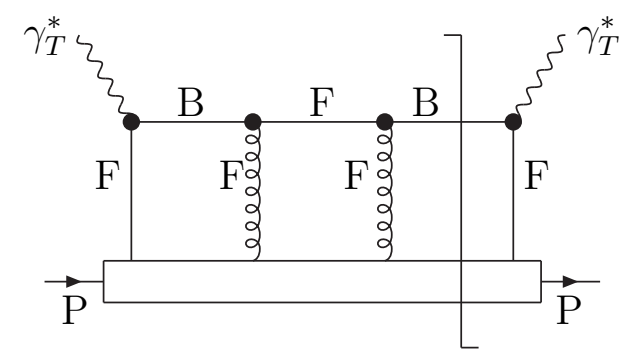

(a)

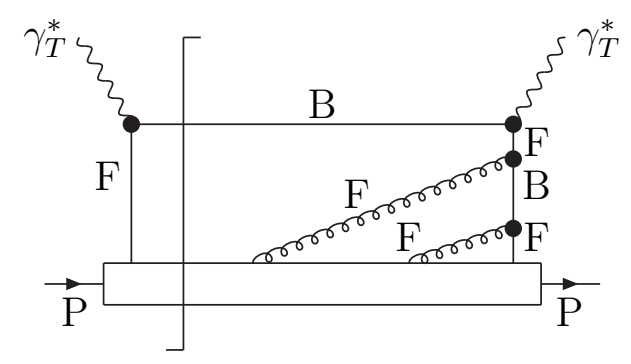

(b)

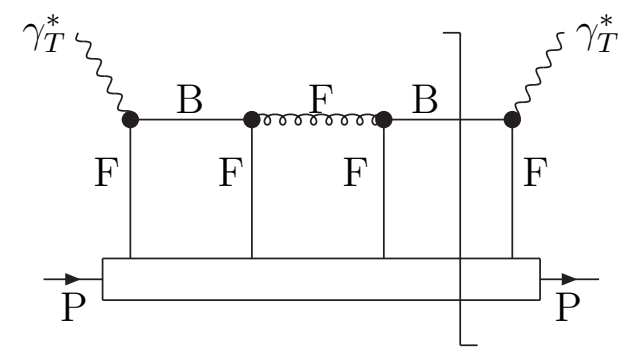

(c)

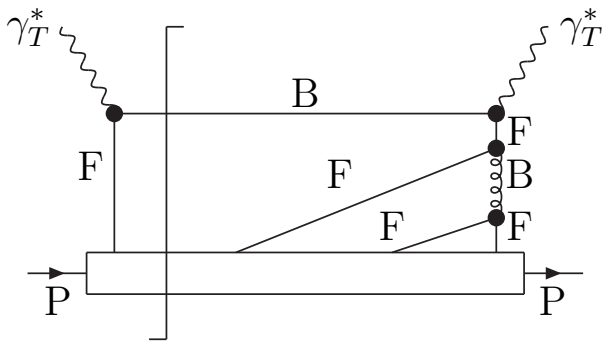

(d)

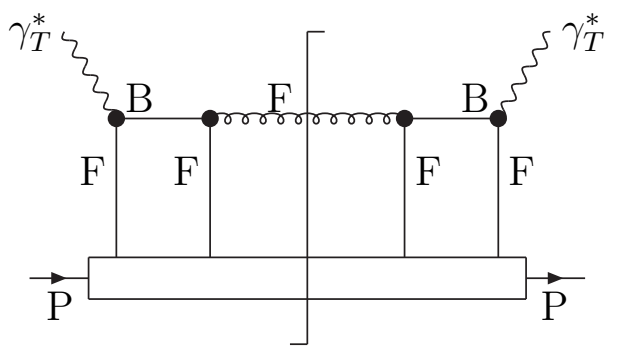

(e)

Fig.3 


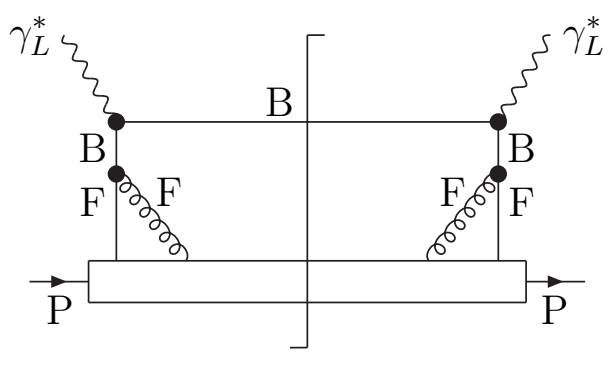

(a)

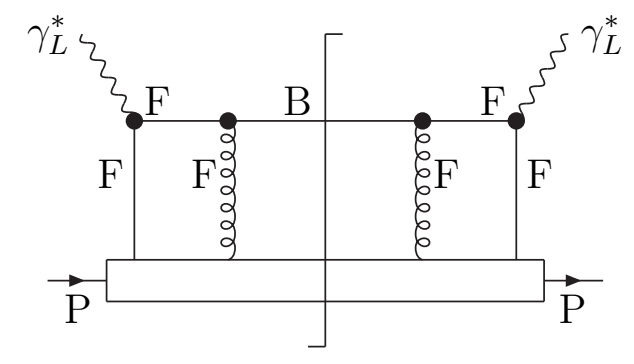

(b)

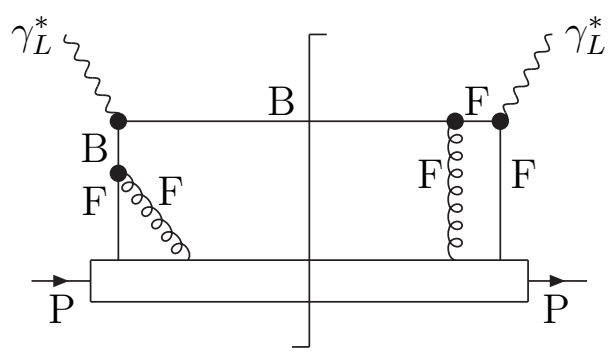

(c)

Fig.4 


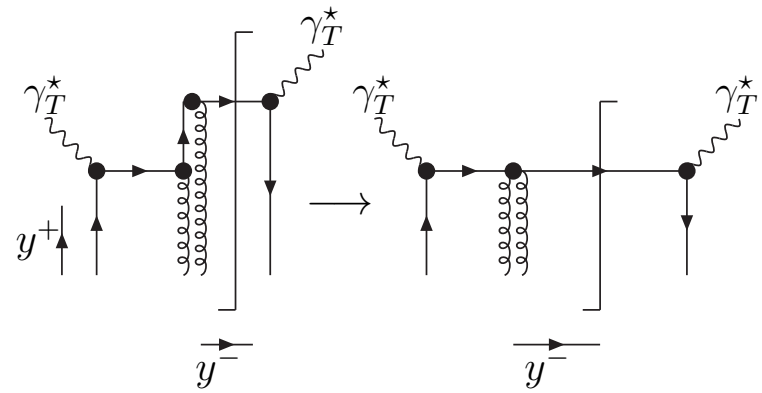

(a)

(b)

Fig.5 


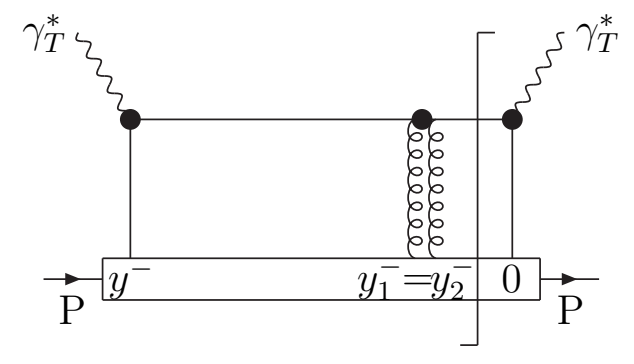

(a)

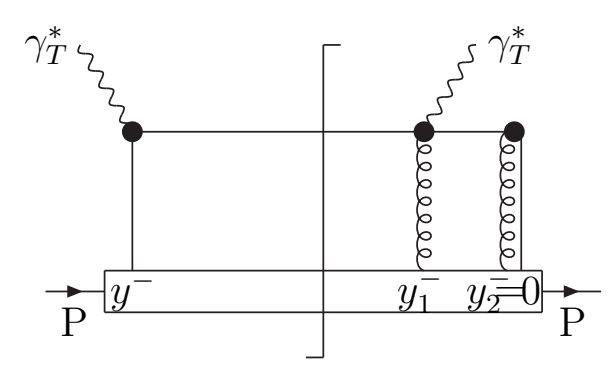

(b)

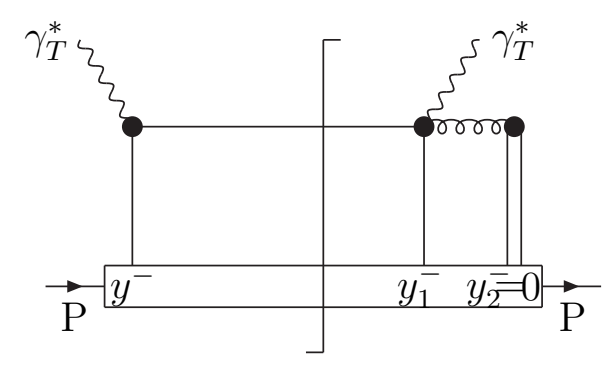

(d)

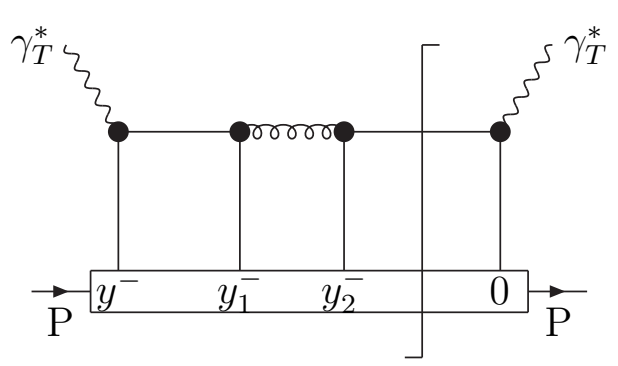

(c)

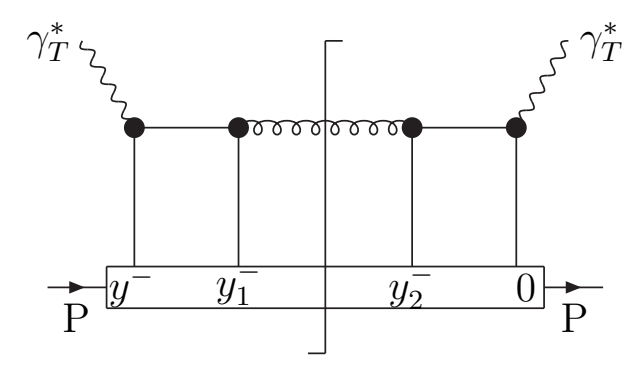

(e)

Fig.6 


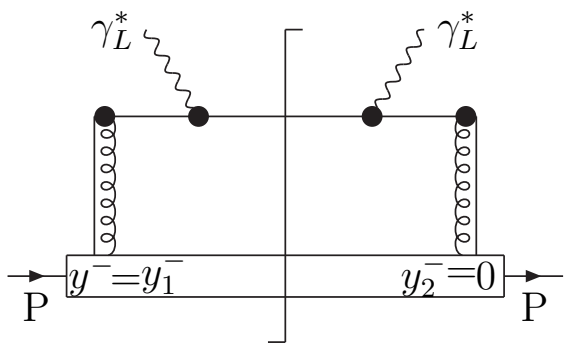

(a)

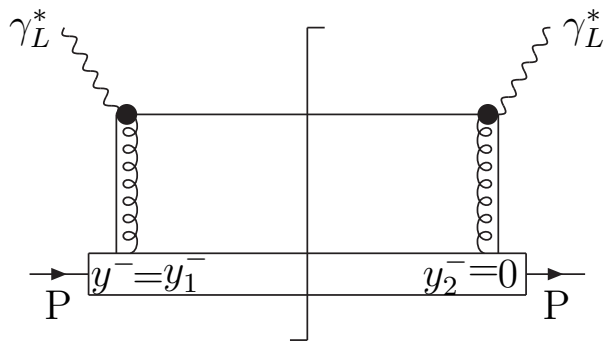

(b)

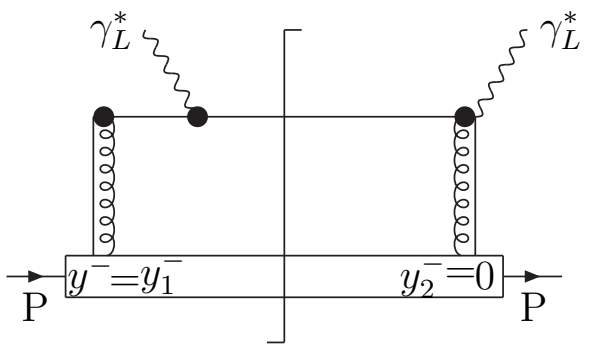

(c)

Fig.7 


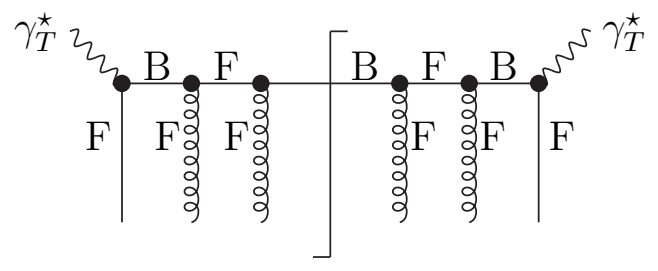

(a)

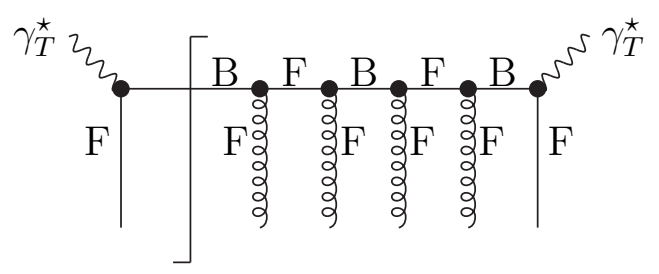

(b)

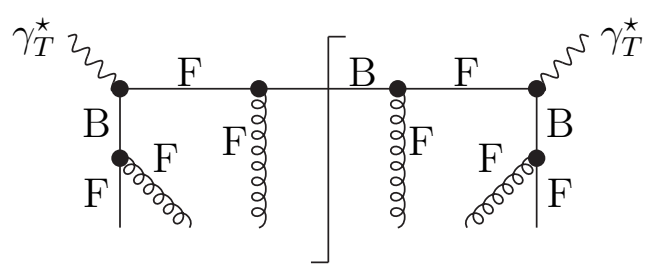

(c)

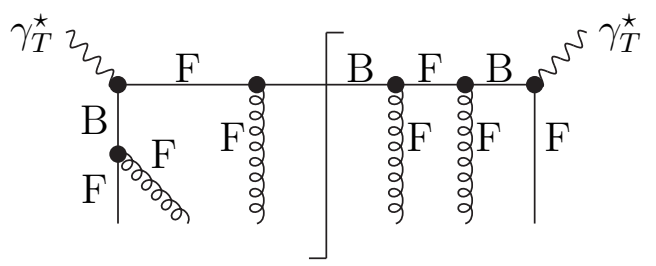

(d)

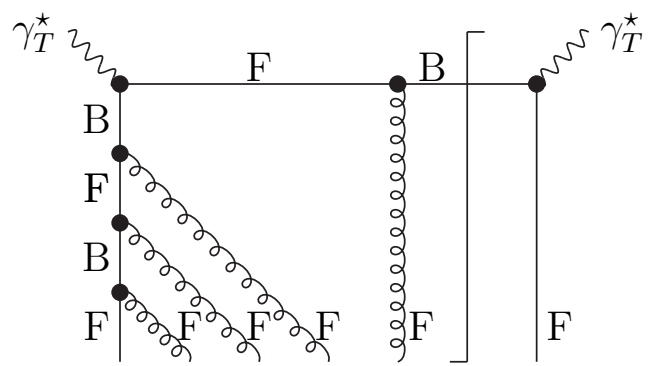

(e)

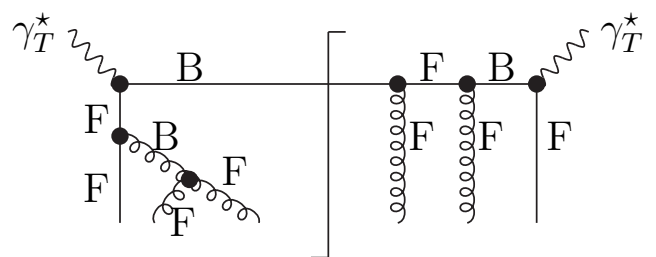

(f)

Fig.8 


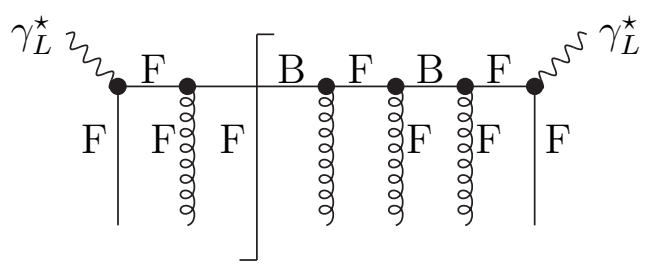

(a)

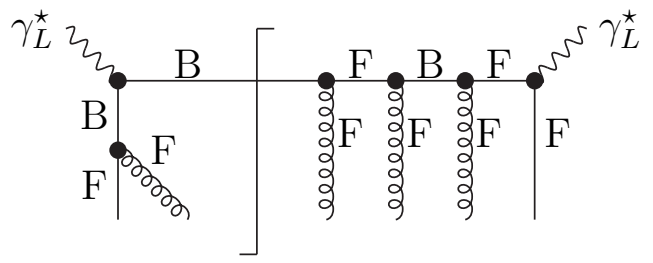

(c)

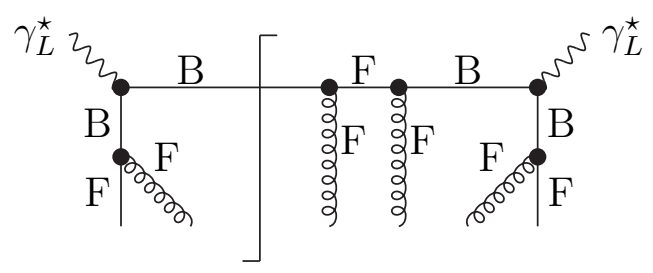

(b)

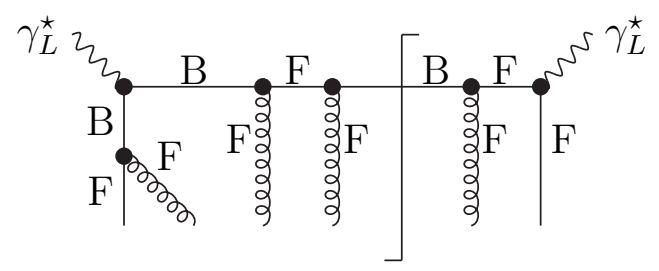

(d)

Fig.9 


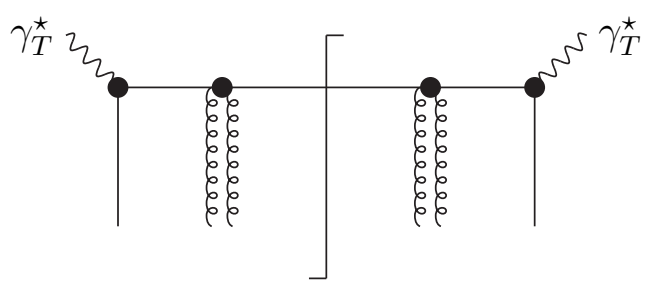

(a)

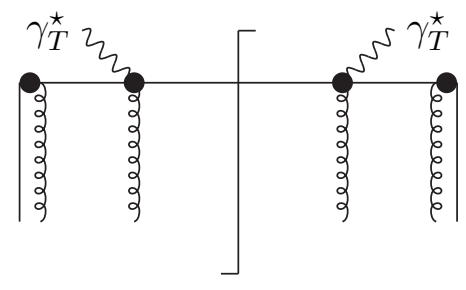

(c)

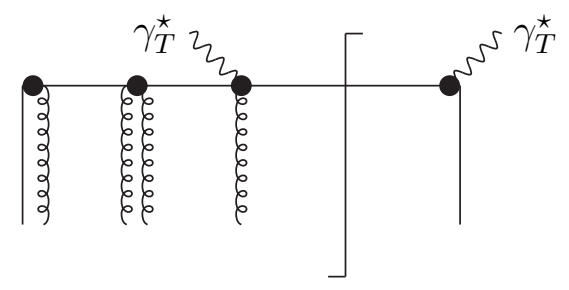

(e)

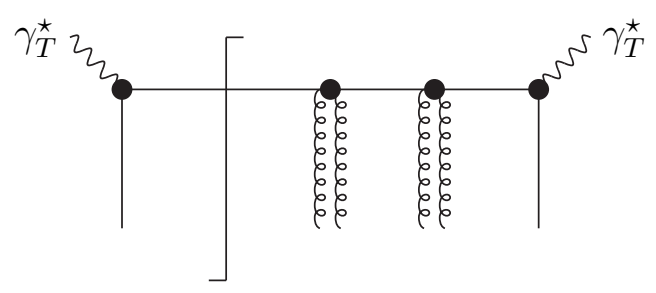

(b)

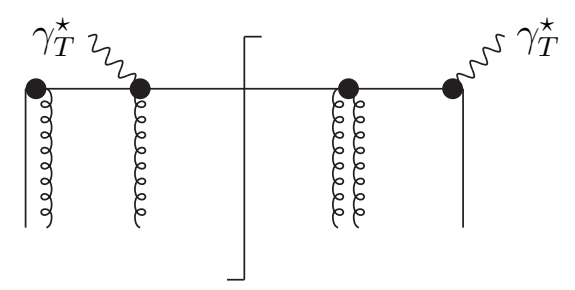

(d)

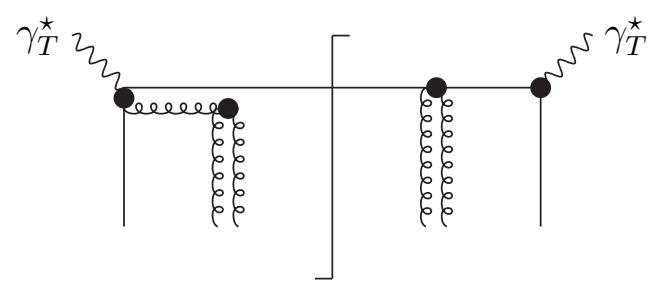

(f)

Fig.10 


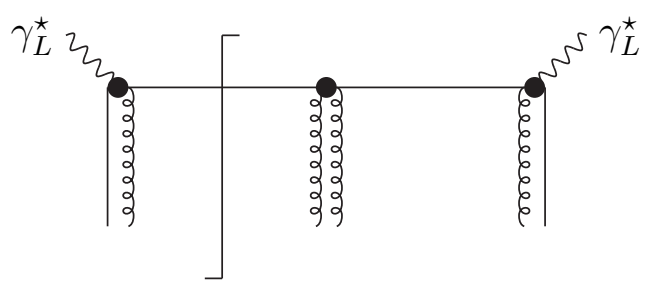

(a)

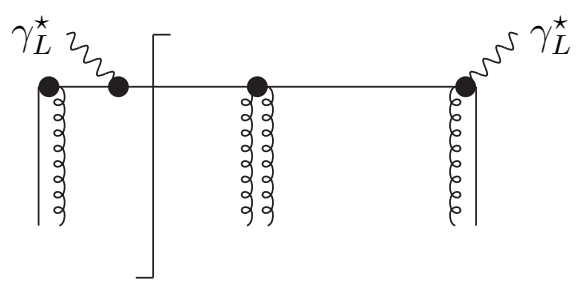

(c)

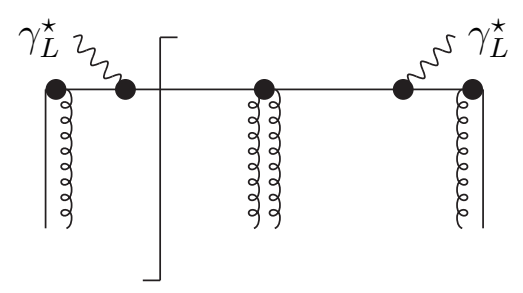

(b)

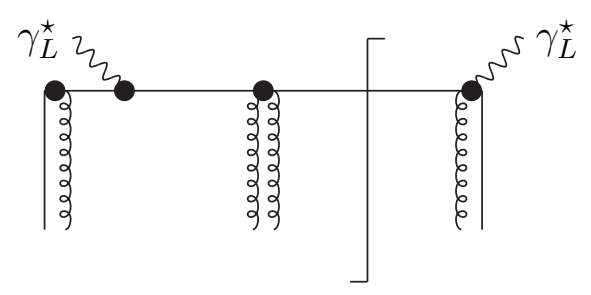

(d)

Fig.11 


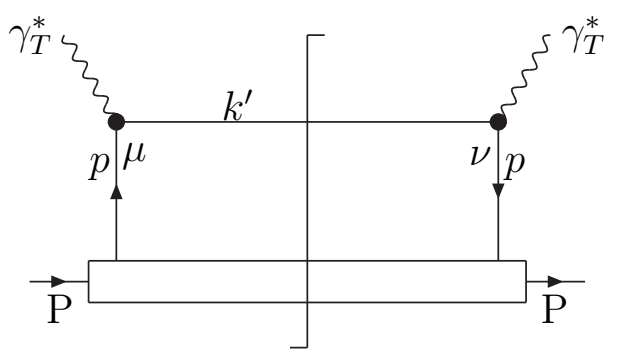

Fig.12 


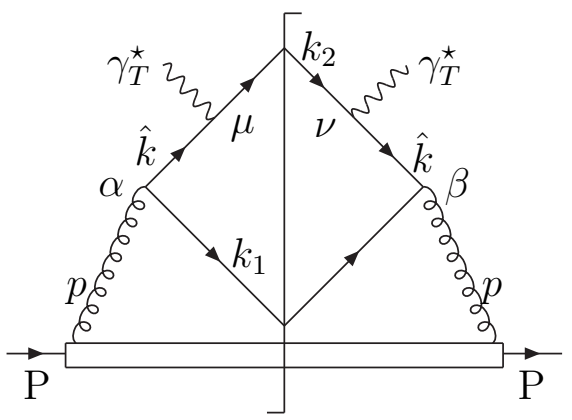

Fig. 13 


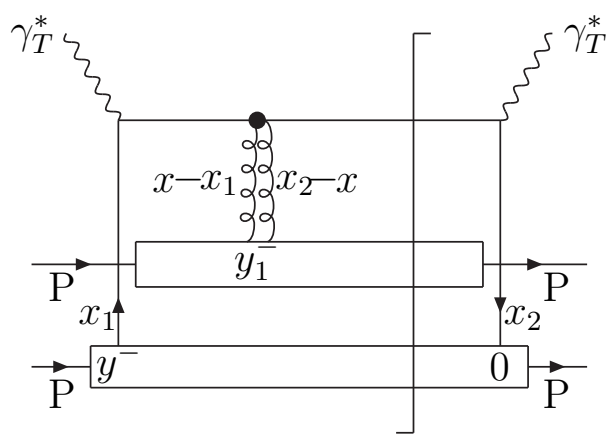

a

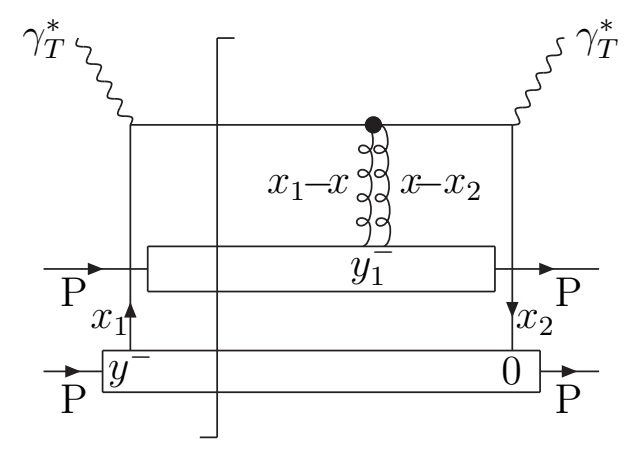

b

Fig.14 


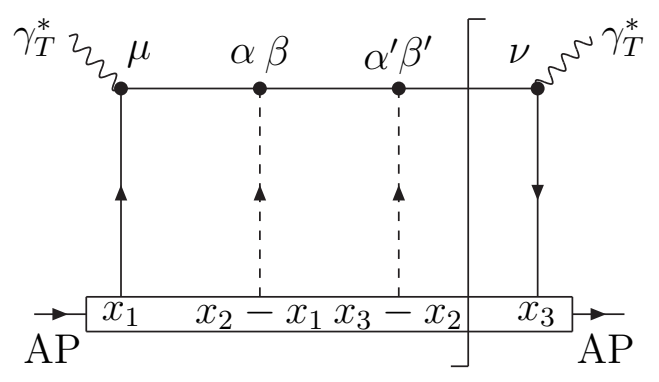

(a)

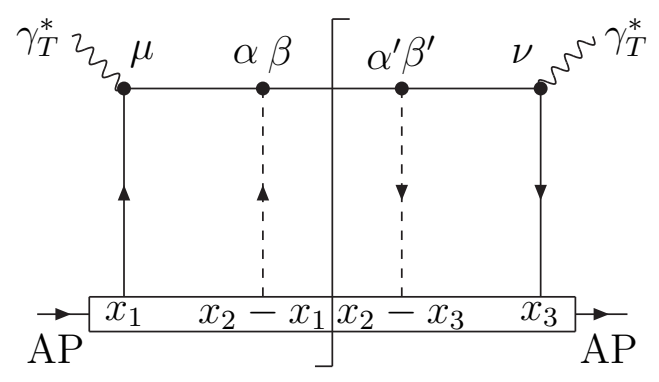

(b)

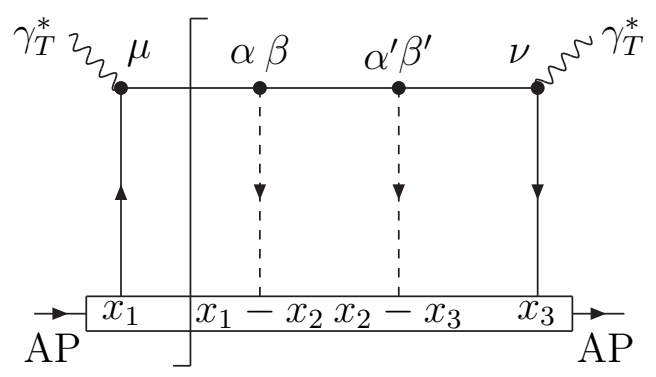

(c)

Fig. 15 


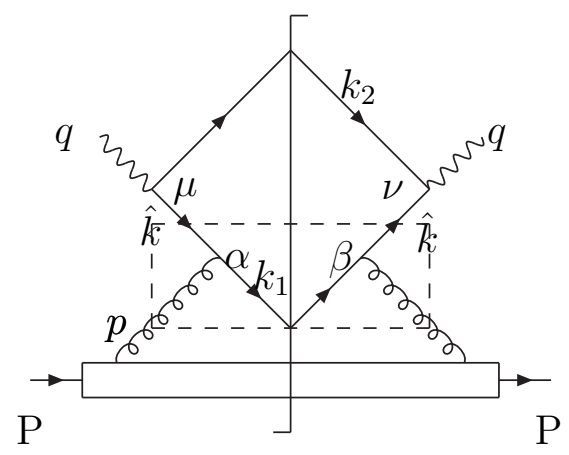

(a)

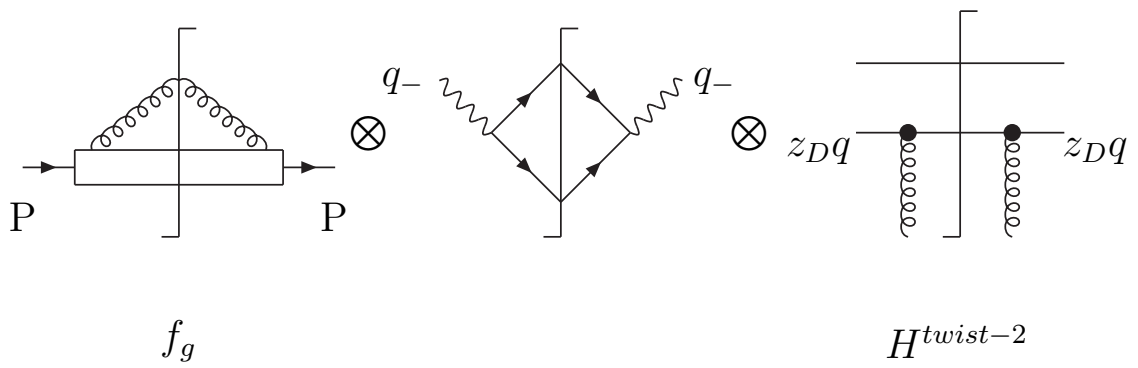

(b)

Fig.16 


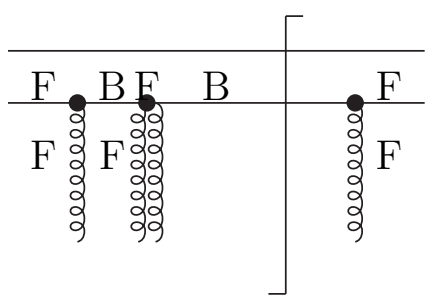

(a)

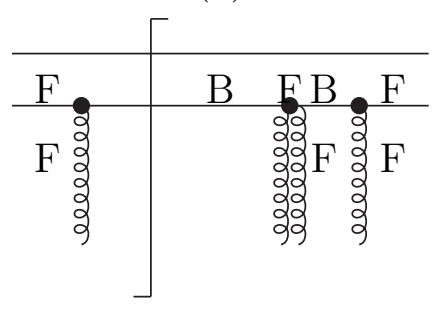

(b)

Fig.17 


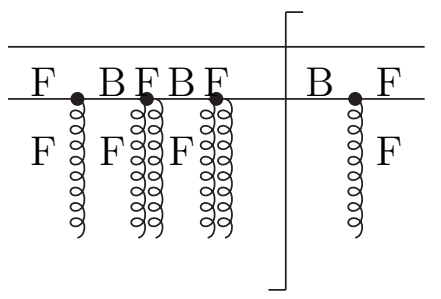

(a)

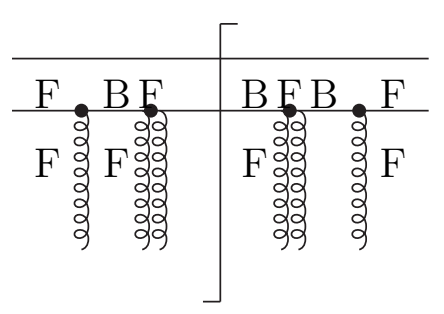

(b)

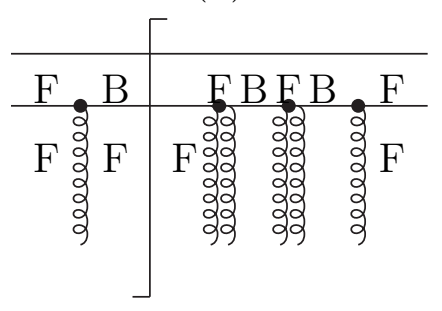

(c)

Fig. 18 


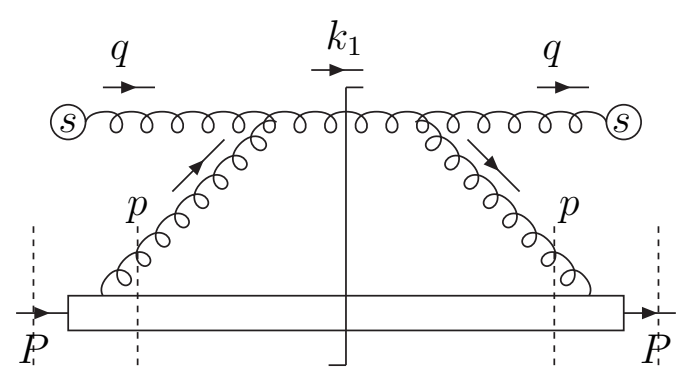

(a)

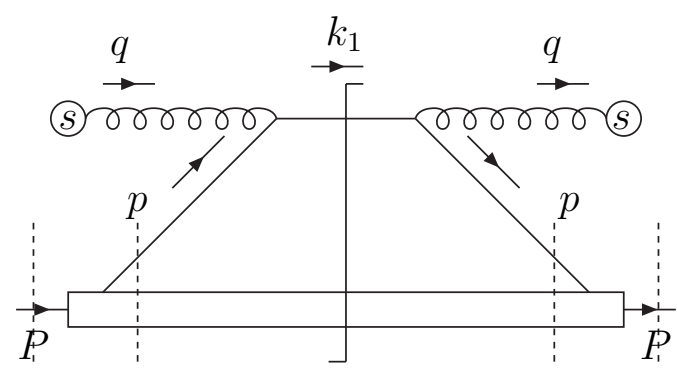

(c)

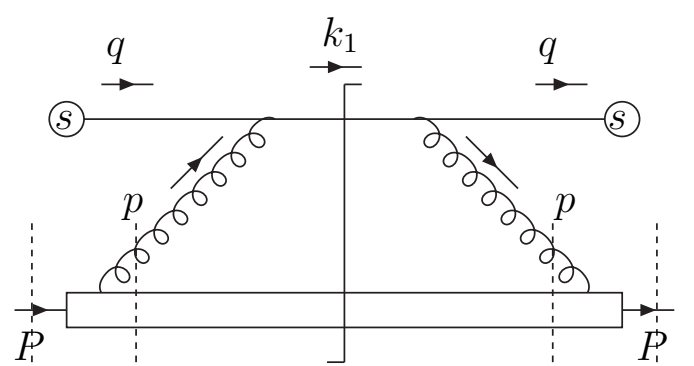

(b)

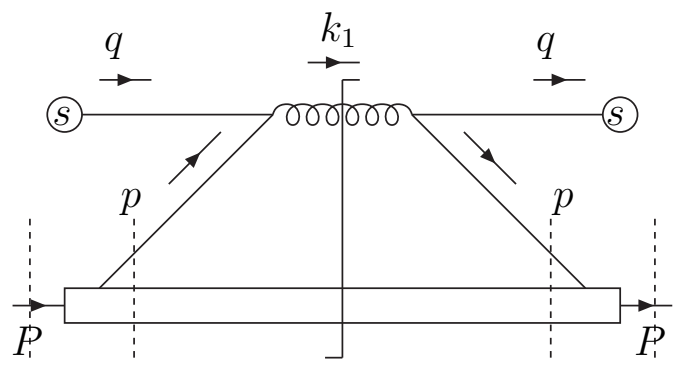

(d)

Fig.19 


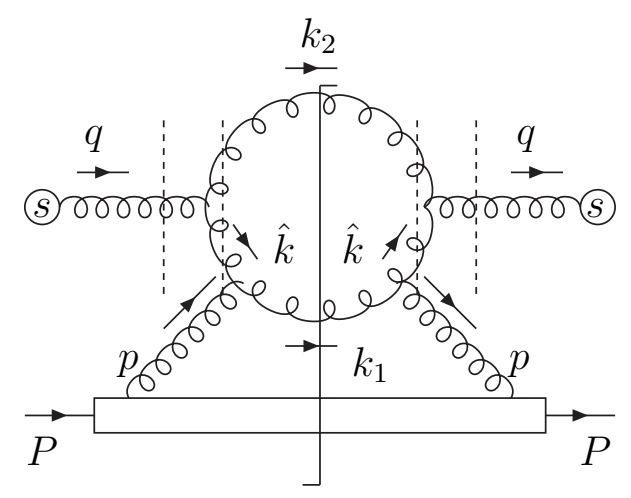

$\left(a^{\prime}\right)$

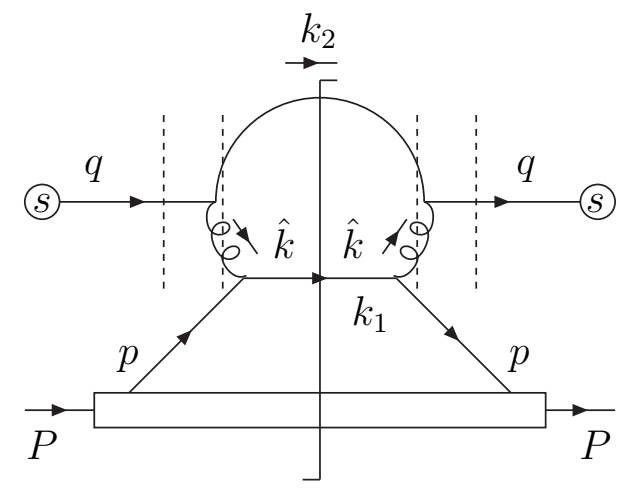

$\left(c^{\prime}\right)$

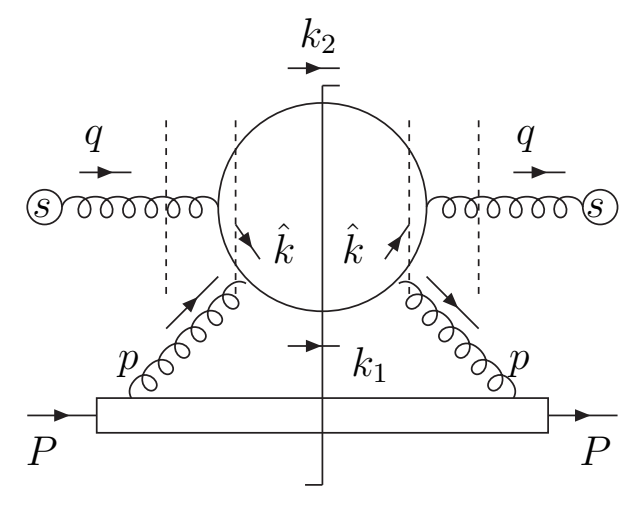

$\left(b^{\prime}\right)$

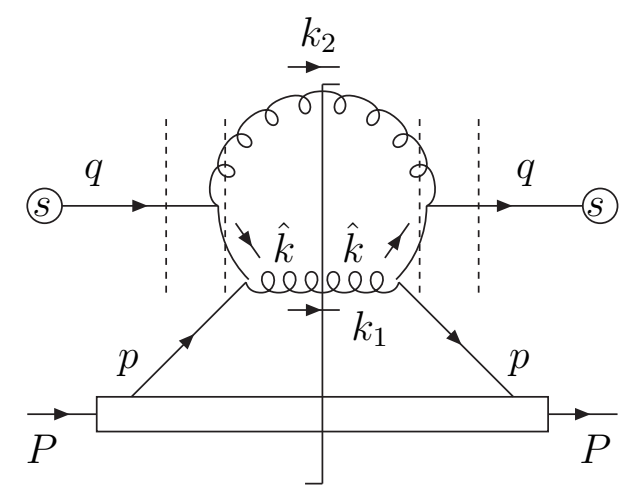

$\left(d^{\prime}\right)$

Fig.20 

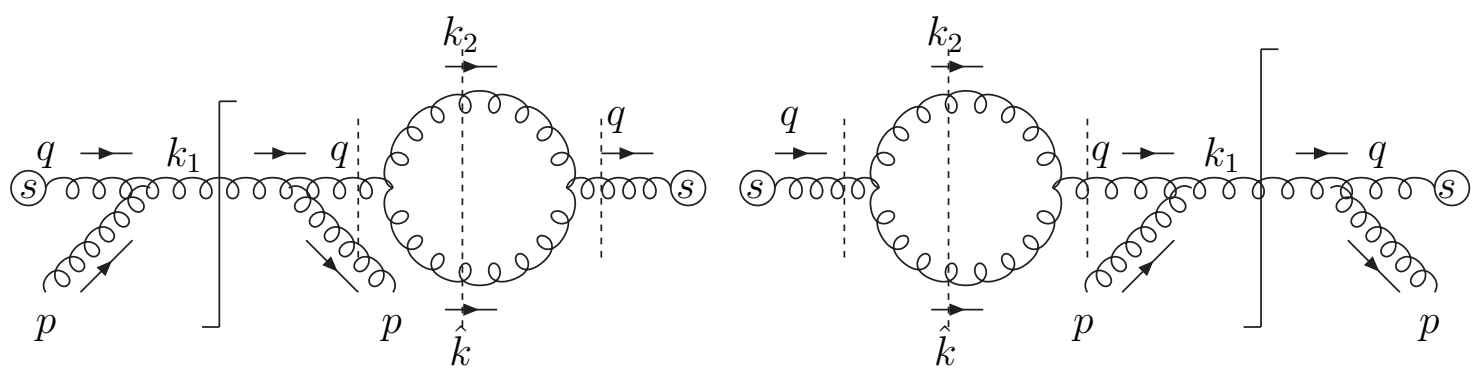

Fig.21 